\title{
ARTICLE
}

\section{A heavy metal P-type ATPase OsHMA4 prevents copper accumulation in rice grain}

Xin-Yuan Huang ${ }^{1, \star}$, Fenglin Deng ${ }^{2, \star}$, Naoki Yamaji ${ }^{2}$, Shannon R.M. Pinson ${ }^{3}$, Miho Fujii-Kashino ${ }^{2}$, John Danku', Alex Douglas ${ }^{1}$, Mary Lou Guerinot ${ }^{4}$, David E. Salt ${ }^{1} \&$ Jian Feng $\mathrm{Ma}^{2}$

Rice is a major source of calories and mineral nutrients for over half the world's human population. However, little is known in rice about the genetic basis of variation in accumulation of copper (Cu), an essential but potentially toxic nutrient. Here we identify OsHMA4 as the likely causal gene of a quantitative trait locus controlling $\mathrm{Cu}$ accumulation in rice grain. We provide evidence that OsHMA4 functions to sequester $\mathrm{Cu}$ into root vacuoles, limiting $\mathrm{Cu}$ accumulation in the grain. The difference in grain $\mathrm{Cu}$ accumulation is most likely attributed to a single amino acid substitution that leads to different OsHMA4 transport activity. The allele associated with low grain $\mathrm{Cu}$ was found in 67 of the 1,367 rice accessions investigated. Identification of natural allelic variation in OsHMA4 may facilitate the development of rice varieties with grain $\mathrm{Cu}$ concentrations tuned to both the concentration of $\mathrm{Cu}$ in the soil and dietary needs.

\footnotetext{
${ }^{1}$ Institute of Biological and Environmental Sciences, University of Aberdeen, Aberdeen AB24 3UU, UK. ${ }^{2}$ Institute of Plant Science and Resources, Okayama University, Kurashiki 710-0046, Japan. ${ }^{3}$ USDA-ARS Dale Bumpers National Rice Research Center, Stuttgart, Arkansas 72160, USA. ${ }^{4}$ Department of Biological Sciences, Dartmouth College, Hanover, New Hampshire 03755, USA. * These authors contributed equally to this work. Correspondence and requests for materials should be addressed to D.E.S. (email: david.salt@abdn.ac.uk) or to J.F.M. (email: maj@rib.okayama-u.ac.jp).
} 
C opper $(\mathrm{Cu})$ is an essential micronutrient for all living organisms. In plants, $\mathrm{Cu}$ acts as a redox-active cofactor and participates in multiple biological processes such as photosynthesis, respiration, cell wall remodelling, oxidative stress resistance and ethylene perception ${ }^{1,2}$. Cu deficiency reduces growth rates, seed set and yield due to impaired photosynthesis and pollen fertility. $\mathrm{Cu}$ also plays important roles in human health as an enzymatic cofactor involved in cellular respiration, free radical detoxification, pigmentation, neuron development, connective tissue formation and iron transport ${ }^{3,4}$. Currently, more than two billion people worldwide suffer from micronutrient deficiencies such as iron $(\mathrm{Fe})$, zinc $(\mathrm{Zn})$ and $\mathrm{Cu}^{5,6}$. Cu deficiency causes immune defects and anaemia ${ }^{3,7}$. The estimated average requirement for $\mathrm{Cu}$ is $260-685 \mu \mathrm{g}$ per day for children depending on age, $700 \mu \mathrm{g}$ per day for adults and $1,000 \mu \mathrm{g}$ per day for women during pregnancy and lactation ${ }^{8}$. In the human diet, $\mathrm{Cu}$ is enriched in meat, fish and nuts. However, such food is not usually available to most populations suffering from micronutrients deficiencies. Therefore, biofortification to increase $\mathrm{Cu}$ in staple foods such as rice is one approach to provide the minimum amount of dietary $\mathrm{Cu}$ for these populations.

On the other hand, $\mathrm{Cu}$ is toxic when present in excess, mainly due to its role in generating highly reactive oxygen species that cause cellular damage $\mathrm{e}^{1-3}$. Due to the over use of $\mathrm{Cu}$-containing fungicides, and the release of $\mathrm{Cu}$ in industrial wastewater and from mining activities, $\mathrm{Cu}$ contamination of cultivated soils and irrigation waters has become problematic in certain regions. For example, $\mathrm{Cu}$ is ranked as the fourth most contaminating heavy metal of agricultural lands in China ${ }^{9}$. Thus, it is important to develop rice (Oryza sativa L.) cultivars that are both tolerant to $\mathrm{Cu}$ and that can exclude excess $\mathrm{Cu}$ from the grain.

Because $\mathrm{Cu}$ is both essential and toxic depending on concentration, organisms including plants have developed a finely tuned homoeostatic network to control cellular $\mathrm{Cu}$ concentrations. $\mathrm{Cu}$ homoeostasis in plants depends on the control of root uptake, root-to-shoot translocation, vacuolar compartmentation and distribution/redistribution of $\mathrm{Cu}$ to various organs. In plants, $\mathrm{Cu}$ is mainly taken up in roots by CTR-like high-affinity $\mathrm{Cu}$ transporters (COPT) such as COPT1 in Arabidopsis thaliana ${ }^{10}$ and rice ${ }^{11}$. Several heavy metal P-type ATPases have been shown to function in $\mathrm{Cu}$ homoeostasis in Arabidopsis and rice. AtHMA5 is involved in loading $\mathrm{Cu}$ into the xylem for root-to-shoot translocation and/or $\mathrm{Cu}$ detoxification in roots $^{12,13}$. AtHMA6/PAA1 and AtHMA8/PAA2 are responsible for transporting $\mathrm{Cu}$ into chloroplasts. AtHMA6/PAA1 transports $\mathrm{Cu}$ across the chloroplast envelope, while the thylakoid membrane localized AtHMA8/PAA2 likely transports $\mathrm{Cu}$ into the thylakoid lumen ${ }^{14,15}$. AtHMA7/RAN1 has been proposed to deliver $\mathrm{Cu}$ to ethylene receptors ${ }^{16,17}$. In rice, OsHMA5 has been shown to be involved in loading $\mathrm{Cu}$ to the xylem for root-toshoot translocation ${ }^{18}$. A yellow stripe-like protein, YSL16, is required for recycling $\mathrm{Cu}$ from older tissues to the young developing tissues as well as grains ${ }^{19}$. However, many transporters involved in other $\mathrm{Cu}$ transport processes remain unidentified.

We previously identified 134 quantitative trait loci (QTL) that control variation in the concentration of 16 elements (P, Mg, K, S, $\mathrm{Ca}, \mathrm{As}, \mathrm{Cd}, \mathrm{Co}, \mathrm{Cu}, \mathrm{Fe}, \mathrm{Mn}, \mathrm{Mo}, \mathrm{Ni}, \mathrm{Rb}, \mathrm{Sr}$ and $\mathrm{Zn}$ ) in unmilled rice grain using two synthetic rice mapping populations ${ }^{20}$. On the basis of a recombinant inbred population derived from a cross between Lemont (LM, japonica) and TeQing (TQ, indica; LT-RILs), and a TeQing-into-Lemont backcross introgression lines (TILs) population, 12 QTLs controlling grain $\mathrm{Cu}$ concentration were identified in either one or both mapping populations under flooded and unflooded field conditions ${ }^{20}$. Among them a major QTL for grain $\mathrm{Cu}$ was detected on chromosome 2 (designated qGCu2-1), which explains up to $43 \%$ of variation in grain $\mathrm{Cu}$ in the LT-RILs. In this study, we identify the gene most likely responsible for this QTL. We find that qGCu2-1 likely encodes a heavy metal $\mathrm{P}_{1 \mathrm{~B}}$-type ATPase, OsHMA4. OsHMA4 localizes to the vacuolar membrane of root cells and we provide evidence that it functions in sequestering $\mathrm{Cu}$ into the vacuoles. Loss-of-function of OsHMA4 results in increased root-to-shoot translocation of $\mathrm{Cu}$, and subsequently increases $\mathrm{Cu}$ accumulation in rice grain. Furthermore, we provide evidence that the genotypic difference in grain $\mathrm{Cu}$ results from different transport activities of OsHMA4 for $\mathrm{Cu}$ due to a single amino acid substitution.

\section{Results}

Map-based cloning of $q$ GCu2-1. To confirm the qGCu2-1 QTL for grain $\mathrm{Cu}$ accumulation we detected previously ${ }^{20}$, we grew the LT-RIL and TIL populations over multiple years under both flooded and unflooded field conditions. This $\mathrm{Cu}$ QTL was consistently detected in grain of both the LT-RILs and TILs, irrespective of year or growth conditions (Fig. 1a,b). Furthermore, qGCu2-1 was also detected in both grain and leaf tissue from TILs cultivated in the greenhouse (Fig. 1b). The reproduction of the qGCu2-1 Cu QTL in greenhouse-cultivated material enabled us to fine map the QTL using greenhouse cultivated plants (Fig. 1b).

To fine map the qGCu2-1 locus, we crossed four LT-RIL lines containing the chromosome fragment from TQ in the mapping region with $\mathrm{LM}$ and generated $\mathrm{F}_{2}$ progeny by self-pollination. Five plants with recombinations between markers $\mathrm{H} 24454$ and $\mathrm{H} 26652$ were isolated from 1,258 $\mathrm{F}_{2}$ plants. These selected $\mathrm{F}_{2}$ plants were self-pollinated and integration of grain $\mathrm{Cu}$ concentration and genotypic data of $\mathrm{F}_{2: 3}$ progeny families narrowed the QTL interval down to a $273 \mathrm{~kb}$ region between the markers RM3294 and RM6378 (Fig. 1c). Among 41 genes in this region (Supplementary Table 1), 2 genes encoding putative heavy metal transporters were identified as candidate genes: a metal cation transporter gene (LOC_Os02g10230, OsZTP29) and a Cu-transporting ATPase gene (LOC_Os02g10290, OsHMA4). OsZTP29 shares $80 \%$ amino acid identity with the zinc transporter ZTP29 in Arabidopsis ${ }^{21}$. Sequence analysis revealed two synonymous single-nucleotide polymorphisms (SNPs) in the coding sequence of OsZTP29 between the LM and TQ cultivars (Fig. 1d). Five SNPs and five small insertions and deletions were found in the promoter sequence of OsZTP29, which did not change its expression level between TQ and LM (Supplementary Fig. 1a), suggesting that OsZTP29 is likely not the casual gene for $q G C u 2-1$. However, comparison of OsHMA4 sequences identified two SNPs in the coding region of OsHMA4 between TQ and LM. Among the two SNPs, only one SNP altered the amino acid sequence, with a valine (V) in TQ and an alanine (A) in LM at amino acid 914 (Fig. 1d). Structure prediction showed that OsHMA4 has eight transmembrane domains and the polymorphic V914A occurs in the seventh transmembrane domain. Homology modelling indicates that V914A localizes in the membranous Cu-binding site I, which includes the conserved YN and CPC motifs ${ }^{22}$ (Fig. 1e,f; Supplementary Fig. 2). A single $\mathrm{SNP}$ in the $3 \mathrm{~kb}$ promoter region was also found between LM and TQ, but this did not alter the expression level of OsHMA4 (Supplementary Fig. 1b) and there is no known cis element at this SNP.

Taking advantage of the existence of residual heterozygosity in TILs, we developed appropriate near isogenic lines (NIL) by generating heterogeneous inbred families (HIFs) (Supplementary Fig. 3 $)^{23,24}$. TIL626 was identified to be heterozygous at the OsHMA4 locus. The HIF626-TQ and HIF626-LM lines were isolated in the next generation. These lines are identical at the 
a

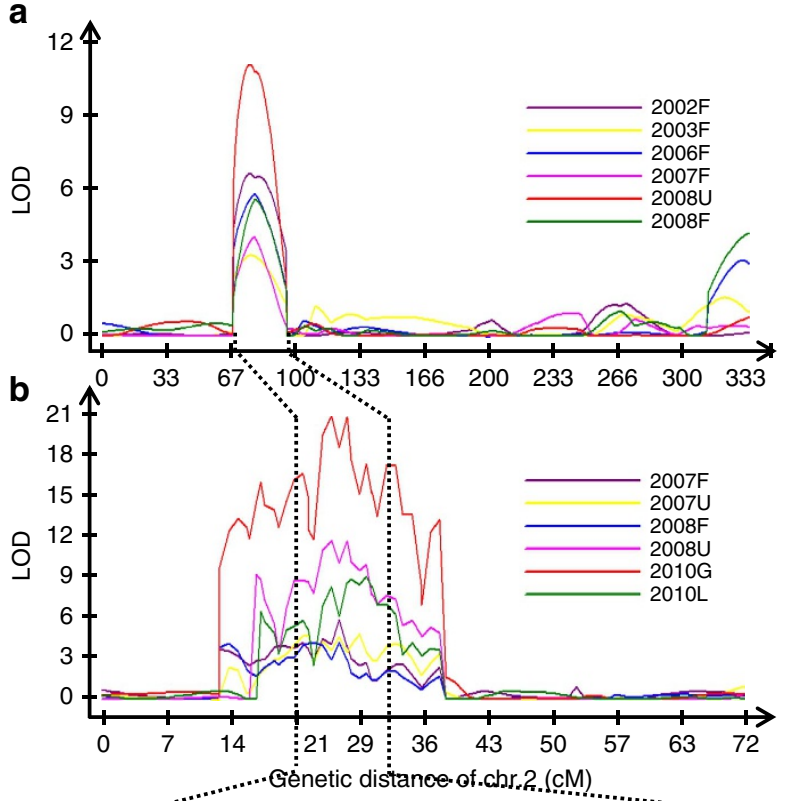

e

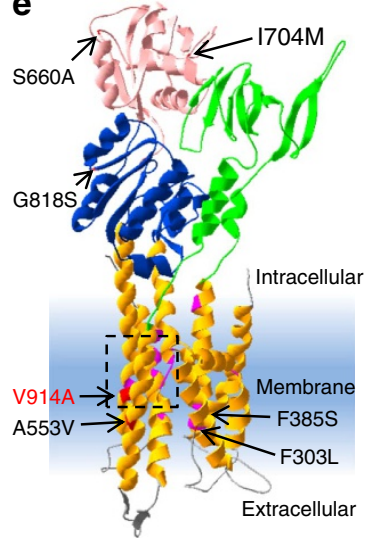

$\mathbf{f}$

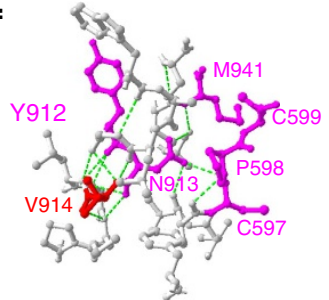

C

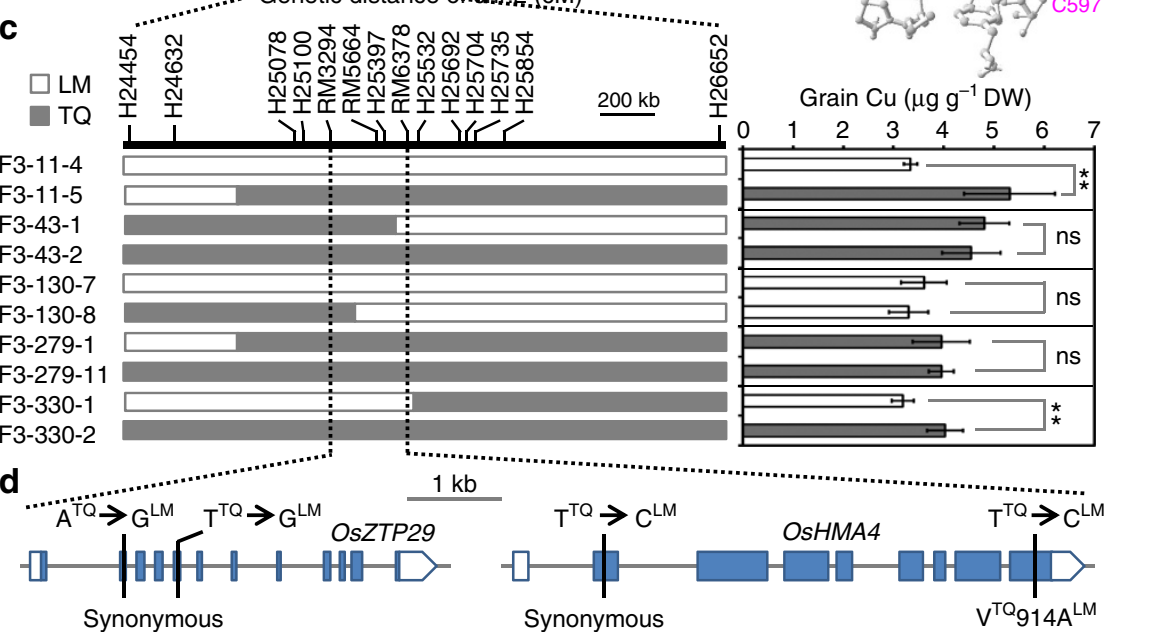

Figure 1 | QTL analysis and map-based cloning of $\mathbf{q G C u 2 - 1 . ~ ( a , b ) ~ T h e ~ L O D ~ p r o f i l i n g ~ o f ~ q G C U 2 - 1 ~ o n ~ c h r o m o s o m e ~} 2$ in the LT-RIL population (a) and TIL population (b) grown in multiple years under different conditions. F, flooded; U, unflooded. G, grains of TILs grown in greenhouse; L, leaves of greenhouse grown TILs. (c) Fine mapping of $q G C u 2-1$. The grain $\mathrm{Cu}$ of homozygous $F_{3}$ plants of five recombinants was determined. Data are presented as mean \pm s.d. with six individual plants. ${ }^{\star \star} P \leq 0.01$ indicates significant difference (Student's $t$-test). NS, no significant difference. (d) Gene structure and sequence variation of two candidate genes OsZTP29 and OsHMA4 between TQ and LM. (e) Three-dimensional structural model of OsHMA4 generated by homology modelling using a Legionella pneumophila P-type ATPase CopA as a template. The actuator domain, phosphorylation domain, nucleotide binding domain and the transmembrane domain are shown in green, blue, red and orange, respectively. The membranous Cu binding residues are shown in magenta.

Polymorphic amino acids from different varieties are shown and the polymorphic V914A between TQ and LM is highlighted in red. (f) Close-up structure of the region with polymorphic V914A. The YN and CPC motif are shown in magenta. The green dash lines indicate H-bonds.

majority of loci in the genome and only differ in a small genomic region containing homozygous OsHMA4 alleles from TQ or LM, respectively (Supplementary Fig. 3). Elemental analysis showed that HIF626-TQ accumulated significantly higher concentrations of $\mathrm{Cu}$ in both grain and leaf than HIF626-LM (Fig. 2a), suggesting that the TQ allele contributes to high $\mathrm{Cu}$ in the grain. This is consistent with the prior QTL mapping results, where TQ was found to contribute the $q G C u 2-1$ allele for higher grain $\mathrm{Cu}^{20}$.

Phenotypic analysis of oshma4 knockout mutant. To test whether OsHMA4 has a biological role consistent with being the causal gene for $q G C u 2-1$, we obtained a T-DNA insertion mutant of OsHMA4. The T-DNA insertion in the fifth intron of OsHMA4 completely interrupts its expression (Supplementary Fig. $4 \mathrm{a}-\mathrm{c}$ ). The T-DNA oshma4 mutant is slightly shorter and has lower fertility compared to the wild-type (WT; Supplementary Fig. 5a-f). Elemental analysis showed that the $\mathrm{Cu}$ concentration in the grain of oshma4 was $138 \%$ higher than that of the WT $(P<0.001$, Student's $t$-test, $n=12)$ (Fig. $2 \mathrm{~b})$. Of the 22 elements measured, $\mathrm{Cu}$ is the only element that showed a major change in concentration in the grain of oshma4, suggesting a specific effect of OsHMA4 on $\mathrm{Cu}$ (Fig. 2b). We also only observed a significant difference in grain $\mathrm{Cu}$ concentration between HIF626-TQ and HIF626-LM (Fig. 2b).

To further assess whether OsHMA4 may be the causal gene for qGCu2-1, we crossed oshma4 and WT with HIF626-TQ and HIF626-LM, respectively. The $\mathrm{Cu}$ concentration in grain of oshma4 $\times$ HIF626-TQ F1 and oshma4 $\times$ HIF626-LM F1 plants were significantly lower than that of the oshma4 mutant (Fig. 2c), similar to the level in the grains of HIF626-TQ and HIF626-LM, respectively, suggesting that both the TQ and LM OsHMA4 alleles 
a

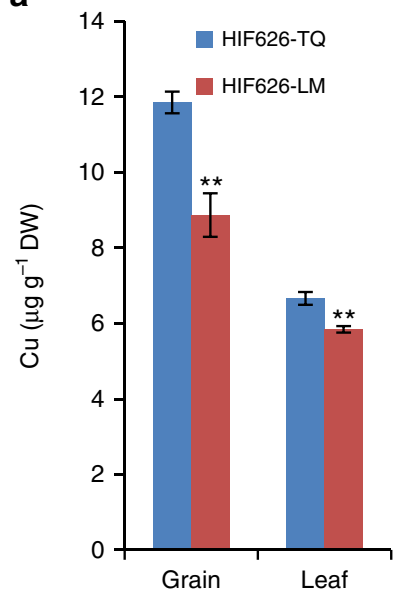

b

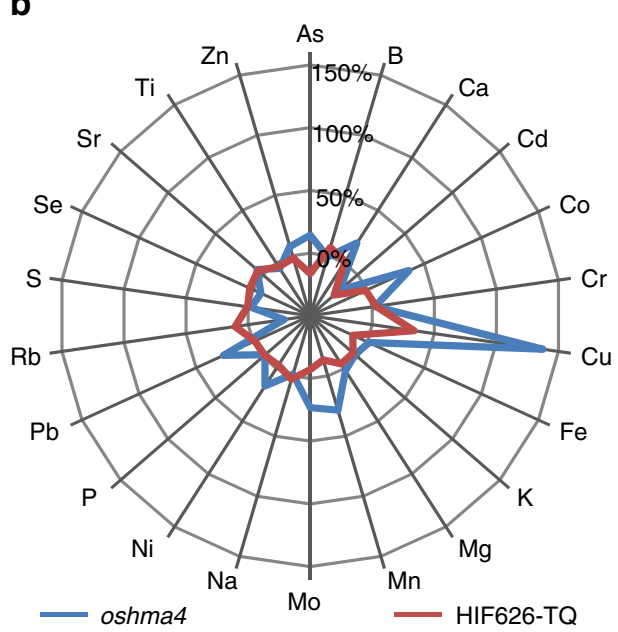

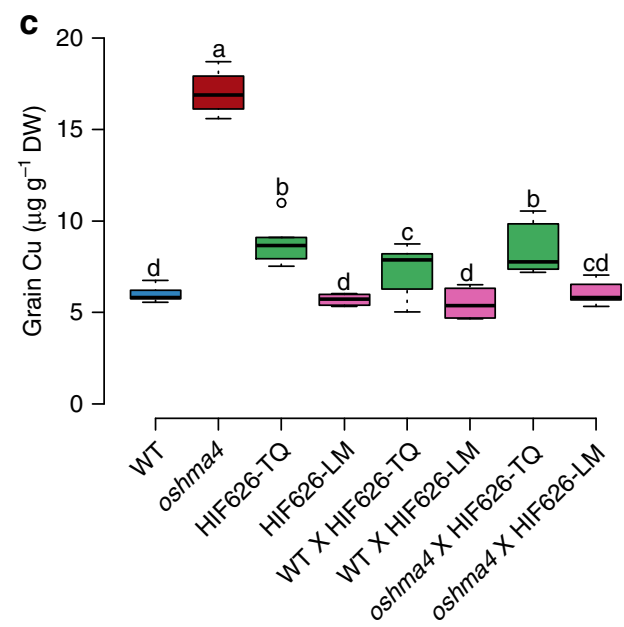

d

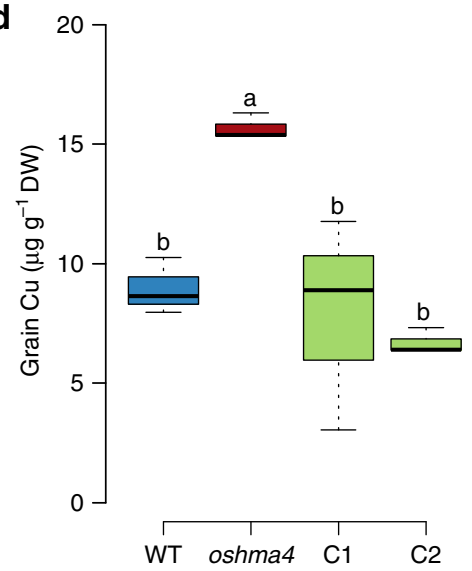

e

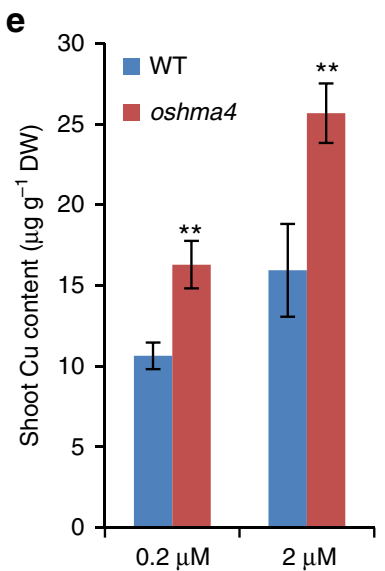

f

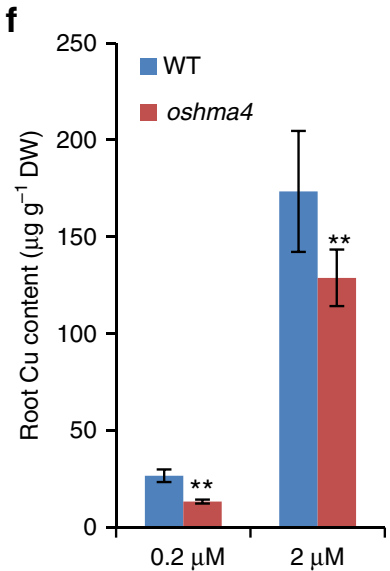

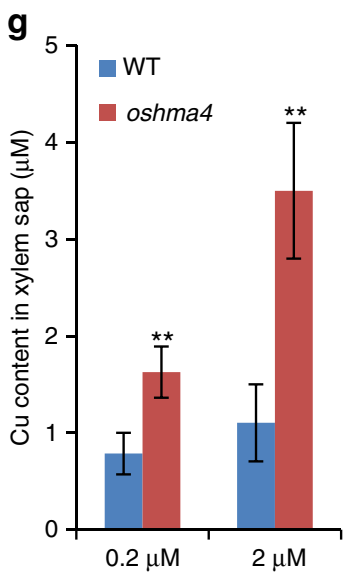

h
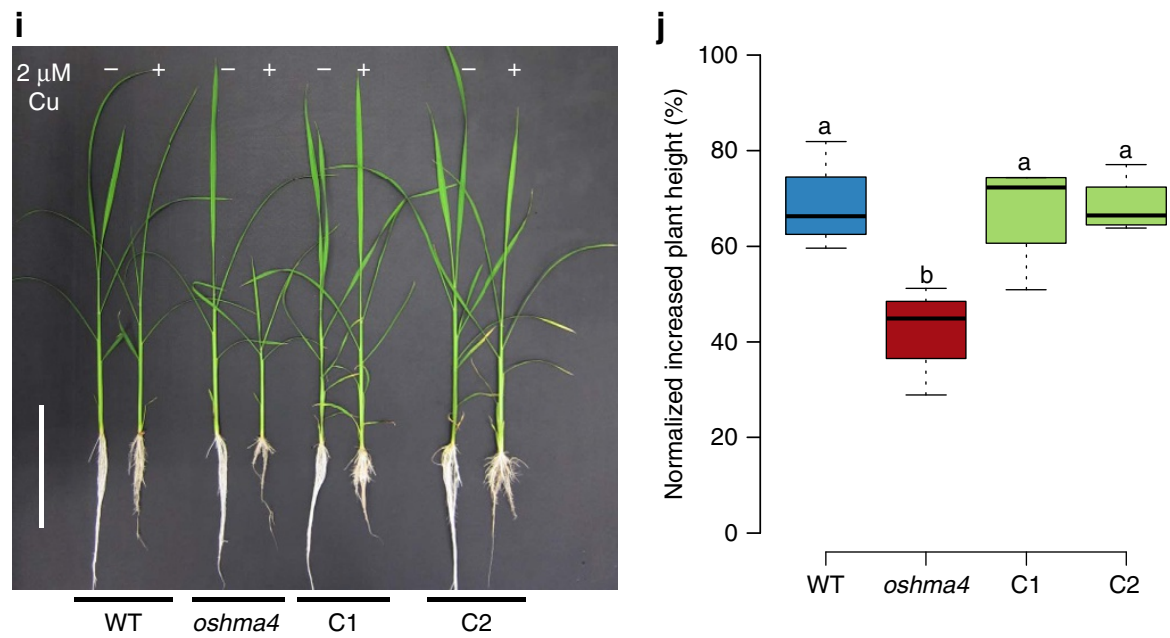

Figure 2 | Characterization of the oshma4 mutant and complementation test. (a) The Cu concentration in the grain and leaf of HIF626-TQ and HIF626-LM. (b) Percentage difference of 22 elements in the grain of oshma4 compared with the WT, and HIF626(TQ) compared with HIF626(LM). Data are visualized in the radar chart. (c) Genetic complementation of oshma4 by crossing with HIF626-TQ or HIF626-LM. The Cu concentration in the grain of F1 plants was determined. (d) Transgenic complementation of oshma4 by transforming Nipponbare cDNA into the oshma4 mutant. Two independent complemented lines are shown. (e-h) The Cu concentration in the shoots (e) and roots (f), xylem sap ( $(\mathbf{g})$ and root cell sap (h). Plants were hydroponically grown in nutrient solution for two weeks and treated with 0.2 or $2 \mu \mathrm{M} \mathrm{CuSO}$ for another week. (i) The image of WT, oshma4 and two complemented lines grown in nutrient solution without (-) or with (+) $2 \mu \mathrm{M} \mathrm{CuSO}$ for 15 days. Bar, $15 \mathrm{~cm}$. (j) The relative shoot growth of plants exposed to $2 \mu \mathrm{M} \mathrm{CuSO}_{4}$ for 15 days. Data in $\mathbf{a}, \mathbf{c}-\mathbf{f}, \mathbf{j}$ are presented as means \pm s.d. ( $n=6$ in $\mathbf{a}, \mathbf{g}, 4-8$ in $\mathbf{c}, 3$ in $\mathbf{d}, 12$ in $\mathbf{e , f}$ and 4 in $\left.\mathbf{h}, \mathbf{j}\right)$. ${ }^{\star} P \leq 0.05$ and ${ }^{*} P \leq 0.01$ indicate significant difference between WT and oshma4 mutant, respectively (Student's $t$-test). Boxes with different letters in $\mathbf{c}, \mathbf{d}, \mathbf{j}$ indicate significant difference at $P \leq 0.05$ (Fisher's least significant difference (LSD) test). DW, dry weight. 
are functional. However, a significant difference in grain $\mathrm{Cu}$ concentration between oshma $4 \times$ HIF626-TQ F1 and oshma $4 \times$ HIF626-LM F1 was observed (Fig. 2c), indicating different functional activity of the TQ and LM OsHMA4 alleles. Furthermore, transfer of a WT DNA fragment containing the OsHMA4 promoter region and the entire open reading frame (ORF) into the oshma4 knockout mutant totally suppressed its high grain Cu phenotype (Fig. 2d; Supplementary Fig. 4d). These results demonstrate that OsHMA4 is indeed responsible for the high grain $\mathrm{Cu}$ phenotype found in oshma4 knockout mutant and both OsHMA4 alleles are functional, although their transport activity is different.

The high $\mathrm{Cu}$ phenotype of the oshma4 mutant was observed not only in the grain but also in the blade and sheath of the flag leaf, as well as the upper nodes and internodes of the main tiller at the harvesting stage (Supplementary Fig. 5g,h). These results indicate that OsHMA4 may not specifically control $\mathrm{Cu}$ in the grain but in all above ground tissues. Further analysis of seedlings grown in nutrient media with either normal or elevated $\mathrm{Cu}$ concentrations showed that oshma4 plants had significantly lower $\mathrm{Cu}$ concentrations in the roots and higher $\mathrm{Cu}$ concentrations in the shoots compared with the WT (Fig. 2e,f). However, there was no difference in other metal concentrations, including $\mathrm{Cd}, \mathrm{Ag}$, Co and $\mathrm{Pb}$ (Supplementary Fig. 6a-e). Cu concentration in the xylem sap was also higher in oshma4 than in the WT (Fig. 2g). Furthermore, the $\mathrm{Cu}$ concentration in the root cell sap, which is mainly composed of the contents of the vacuole ${ }^{25}$, was significantly lower in the oshma4 mutant compared to the WT (Fig. 2h).

We also compared $\mathrm{Cu}$ tolerance between the oshma4 mutant and WT. The oshma4 mutant was more sensitive to elevated $\mathrm{Cu}$ concentrations in the growth media compared with the WT (Fig. 2i,j; Supplementary Fig. 5i). Furthermore, such increased $\mathrm{Cu}$ sensitivity of oshma4 was rescued in the transgenic complementation lines (Fig. 2i,j).

Expression pattern and subcellular localization of OsHMA4. Expression of OsHMA4 was observed in most organs throughout the growth period of Nipponbare grown in a paddy field (Fig. 3a). However, the expression of OsHMA4 was generally much higher in the roots compared with other organs (Fig. 3a). Similar expression pattern was observed in TQ grown in a greenhouse (Supplementary Fig. 7a). Expression of OsHMA4 was strongly induced by high $\mathrm{Cu}$ treatment in the roots but not in the shoots (Fig. 3b,c). OsHMA4 was also slightly induced by $\mathrm{Ag}$ and $\mathrm{Cd}$ treatment but suppressed by $\mathrm{Pb}$ and $\mathrm{Mn}$ treatment (Supplementary Fig. 7b). Under Cu-depleted growth conditions, expression of OsHMA4 was downregulated in the root (Fig. 3d). Such downregulation was also observed under Fe depletion but not under $\mathrm{Zn}$ or $\mathrm{Mn}$ depletion (Supplementary Fig. 7c). The induction by excess $\mathrm{Cu}$ and downregulation by $\mathrm{Cu}$ deficiency suggested a critical role for OsHMA4 in Cu homoeostasis in roots.

Using laser microdissection, we observed that OsHMA4 was mainly expressed in the central cylinder of the mature root (Fig. 3e). To further investigate the tissue-specific localization of OsHMA4, we expressed GFP-OsHMA4 in the OsHMA4 mutant under the control of the native promoter of OsHMA4 (Supplementary Fig. 5j). Immunostaining with an anti-green fluorescent protein (GFP) antibody revealed OsHMA4 to accumulate in the vascular tissues of the stele, mainly in pericycle cells (Fig. 3f). This observation was further supported by $\beta$ glucuronidase (GUS) staining of the OsHMA4 promoter-GUS transgenic rice lines (Supplementary Fig. 7d-f).

Analysis of the subcellular localization of GFP-OsHMA4 after stable heterologous expression in Arabidopsis showed OsHMA4 to be localized to the tonoplast (Fig. 3g). We observed that OsHMA4 from both TQ and LM were localized to the tonoplast, suggesting that the V914A variation has no effect on the subcellular localization of OsHMA4 (Fig. 3g). Subcellular localization of OsHMA4 was further investigated in transgenic rice expressing GFP-OsHMA4 under the control of the native promoter of OsHMA4. Western blot analysis with a GFP antibody showed a single band with the predicted size in the rice transgenic line, but not in WT, indicating the specificity of the GFP antibody (Supplementary Fig. 8a). In situ immunostaining with this GFP antibody further showed that at least part of the signal was localized to the tonoplast, with the immunostaining being observed on the inside facing side of the nuclei (Supplementary Fig. 8c-e). We further showed that the tonoplast localization of OsHMA4 is unlikely to be affected by excess $\mathrm{Cu}$ (Supplementary Fig. 8f). Immunoblotting with GFP antibody of sucrose-density gradient separated microsomal membranes from GFP-OsHMA4 expressing rice roots revealed a weak signal for GFP-OsHMA4 in the tonoplast and plasma membrane (Supplementary Fig. 8b).

Heterologous expression in Arabidopsis and yeast. Sequence analysis revealed that OsHMA4 shares $56.5 \%$ sequence identity with Arabidopsis AtHMA5. AtHMA5 has been shown to be involved in $\mathrm{Cu}$ translocation from roots to shoots and/or $\mathrm{Cu}$ detoxification in roots ${ }^{12,13}$. To investigate whether expression of OsHMA4 in athma 5 could improve its tolerance to excess $\mathrm{Cu}$, we heterologously expressed GFP-OsHMA4 from TQ and LM in athma5 using the $35 \mathrm{~S}$ promoter (Supplementary Fig. 9a). When grown in low $\mathrm{Cu}$, the root elongation of athma5 knockout mutant was similar to that of the WT and transgenic lines carrying GFPOsHMA4 from either TQ or LM in the athma5 background (Fig. 4a,b; Supplementary Fig. 10a,b). However, under high $\mathrm{Cu}$, the root elongation of the athma5 mutant was significantly inhibited, but the introduction of OsHMA4 from either TQ or LM into the athma 5 mutant significantly increased its $\mathrm{Cu}$ tolerance (Fig. 4a,b; Supplementary Fig. 10a,b). Quantification of $\mathrm{Cu}$ showed that the $\mathrm{Cu}$ concentration in athma5 was lower in the shoots and higher in the roots compared with the WT when grown with $50 \mu \mathrm{M} \mathrm{Cu}$. However, in the transgenic lines expressing either allele of OsHMA4, Cu levels in both roots and shoots were similar to that of the athma5 mutant (Fig. 4c,d). These results suggest that although OsHMA4 can suppress the $\mathrm{Cu}$ sensitivity of athma5 this is not achieved by restoring root-toshoot translocation of $\mathrm{Cu}$, but rather most likely by enhancing sequestration of $\mathrm{Cu}$ into root vacuoles. To rule out the ectopic effect of overexpression of OsHMA4 driven by $35 \mathrm{~S}$ promoter, we also expressed OsHMA4 in athma5 using the AtHMA5 native promoter. Expression of the LM OsHMA4 allele from the AtHMA5 native promoter significantly enhanced the resistance of athma 5 to excess $\mathrm{Cu}$, whereas the TQ allele had no consistent affect (Fig. 4a,b; Supplementary Fig. 9b; Supplementary Fig. 10a,b). These results suggest that the TQ OsHMA4 allele is hypofunctional relative to the LM allele.

To investigate whether expression of OsHMA4 in WT Arabidopsis could improve its tolerance to excess $\mathrm{Cu}$, we heterologously expressed GFP-OsHMA4 in Col-0 WT using the $35 \mathrm{~S}$ promoter (Supplementary Fig. 9c). These transgenic lines were more tolerant to $\mathrm{Cu}$ stress compared with non-transgenic WT (Supplementary Fig. 11a-d). Quantification of $\mathrm{Cu}$ showed that under excess $\mathrm{Cu}$, the GFP-OsHMA4 expressing lines accumulated more $\mathrm{Cu}$ in roots than that of WT, further supporting a role for OsHMA4 in sequestration of $\mathrm{Cu}$ into root vacuoles (Supplementary Fig. 11e,f). The expression of $\mathrm{Cu}$ deficiency responsive genes was not markedly affected in the Arabidopsis lines expressing GFP-OsHMA4 with the exception of 

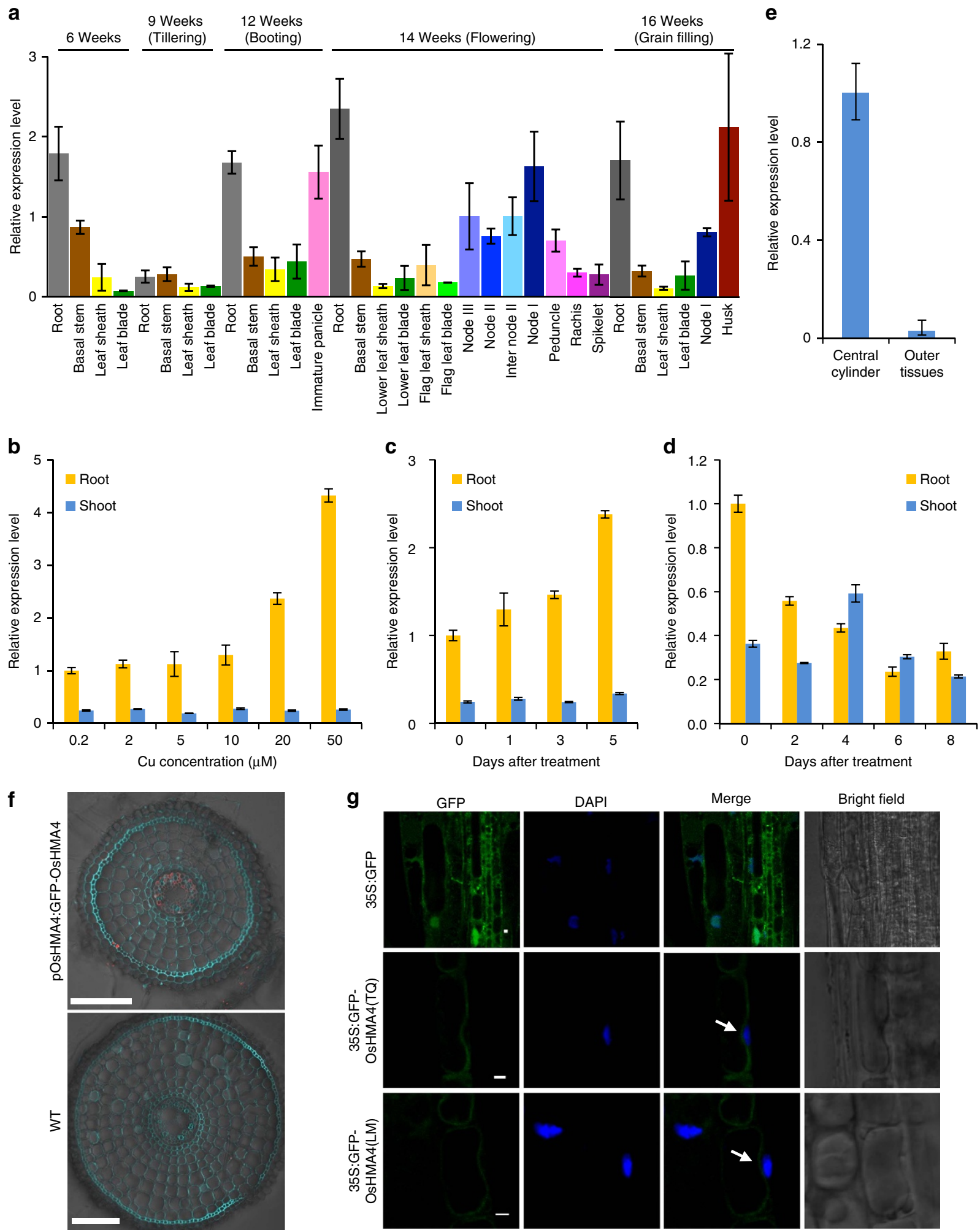

Bright field

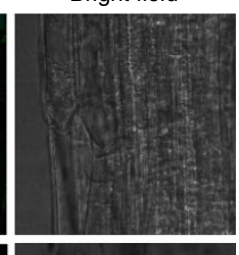

Figure 3 | Expression pattern and subcellular localization of OsHMA4. (a) Expression level of OsHMA4 in different organs at different growth stages Samples were taken from Nipponbare grown in a paddy field. (b) Expression of OsHMA4 in TQ grown in nutrient solution with different concentrations of $\mathrm{Cu}$ for two weeks. (c) Time-course expression of OsHMA4 under Cu treatment. Two-week-old TQ plants were treated with $20 \mu \mathrm{M} C \mathrm{C}$ for indicated days. (d) Expression of OsHMA4 was suppressed under Cu deficiency. TQ plants were grown with $0.2 \mu \mathrm{M} \mathrm{CuSO}_{4}$ for one week and treated with $\mathrm{Cu}$ free nutrient solution for indicated days. Expression level of OsHMA4 was quantified by qRT-PCR. Data were presented as mean \pm s.d. $(n=3)$. (e) OsHMA4 mainly expresses in central cylinder tissue in roots. Tissues were separated by laser microdissection for qRT-PCR. (f) Tissue-specific expression of OsHMA4 in roots. Immunostaining using an antibody against GFP was performed in the root of WT (lower) or transgenic rice expressing GFP-OsHMA4 driven by OsHMA4 native promoter in oshma4 (upper). Red colour indicates the GFP-specific signal. Blue colour indicates cell wall and nucleus stained by 4,6-diamidino-2-phenylindole (DAPI). Bar, $100 \mu \mathrm{m}$. (g) Subcellular localization of OsHMA4 in stable A. thaliana transgenic plants. The nuclei were stained by DAPI. The arrows indicate the vacuolar membrane on the inner side of the nucleus. Bar, $2 \mu \mathrm{m}$. 
a

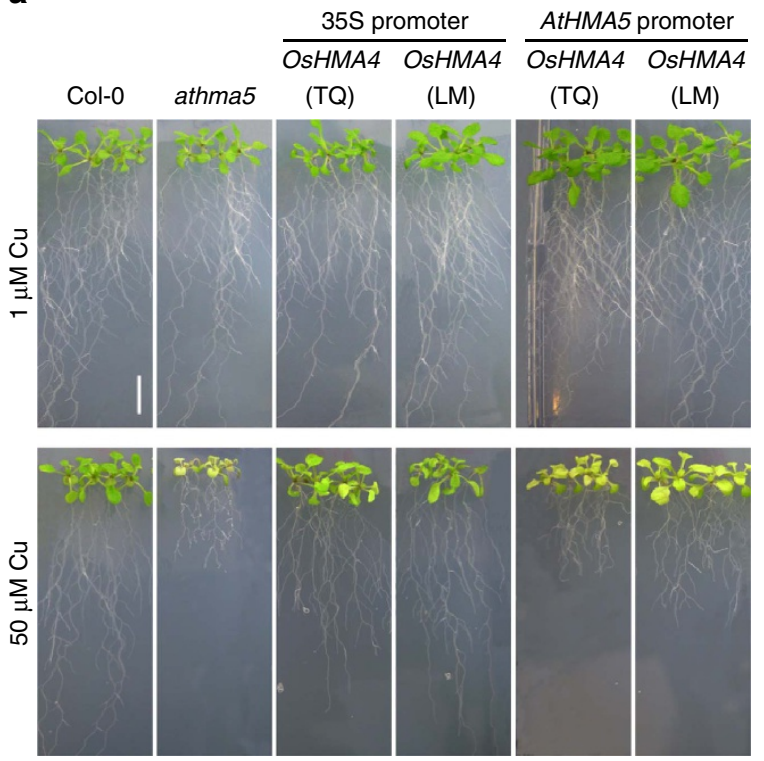

C

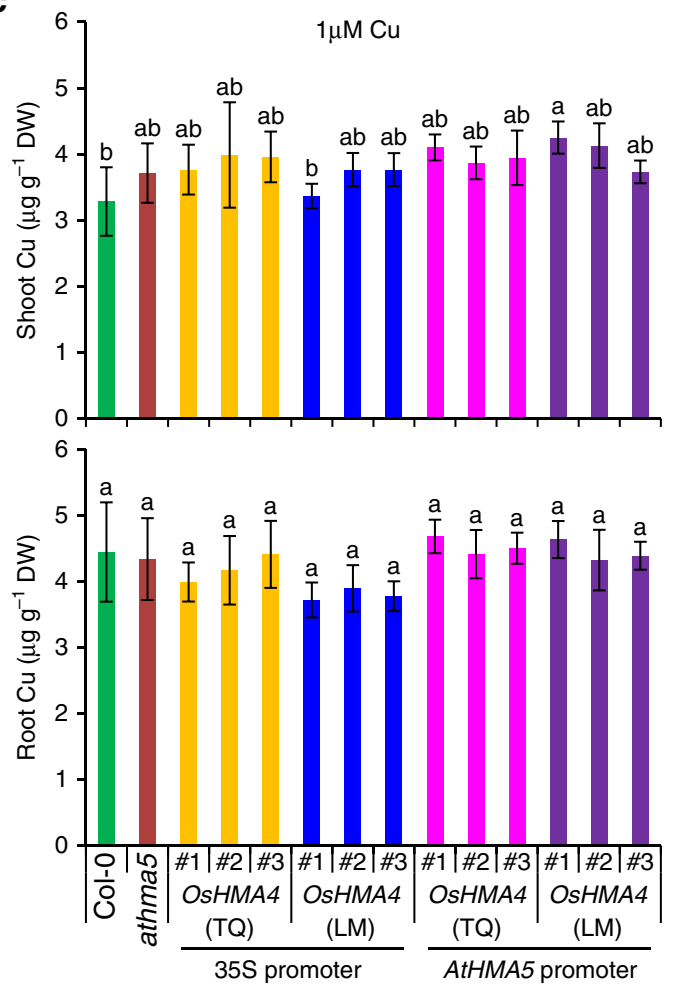

b

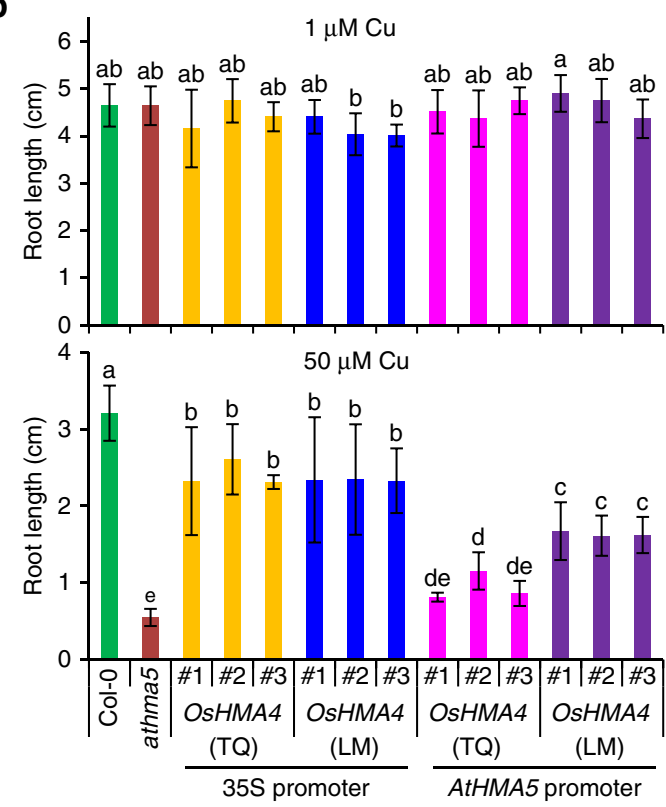

d
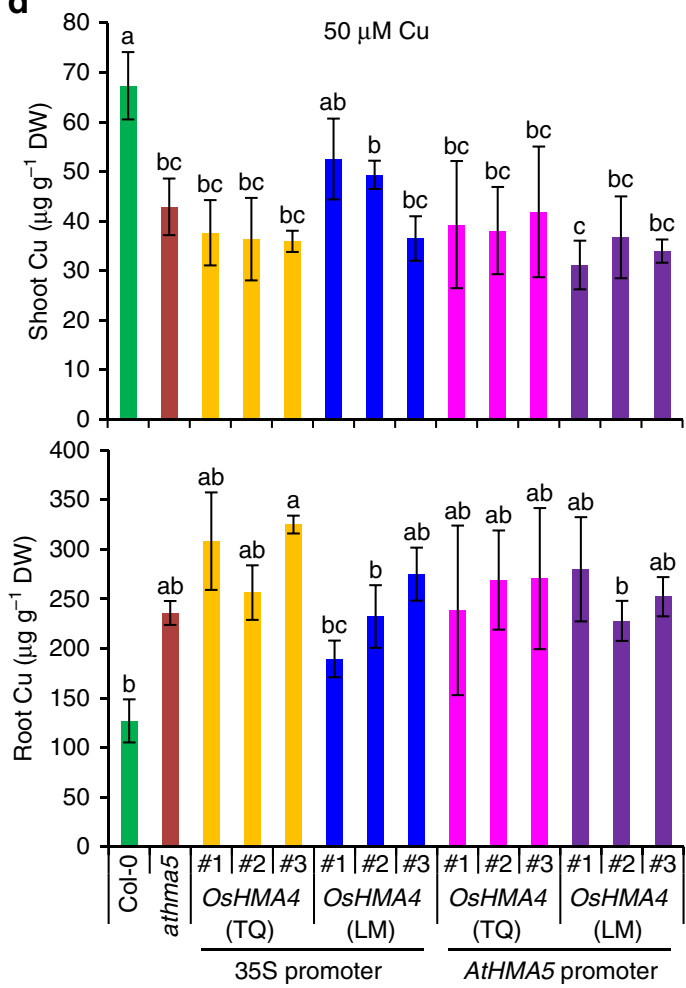

Figure 4 | Expression of OsHMA4 in Arabidopsis athma5 mutant alleviates its Cu sensitive phenotype. (a) Phenotype of athma5 mutant transformed with OsHMA4 from TeQing (TQ) or Lemont (LM) driven by CaMV 35S promoter or AtHMA5 native promoter. Plants were grown on MGRL media with $1 \mu \mathrm{M} \mathrm{CuSO}_{4}$ for 6 days and then transferred to the media containing 1 or $50 \mu \mathrm{M} \mathrm{CuSO}_{4}$ for 10 days. Bar, $1 \mathrm{~cm}$. (b) Root length of OsHMA4 transgenic lines grown on the media containing 1 or $50 \mu \mathrm{M} \mathrm{CuSO}_{4}$. Plants were grown on MGRL media with $1 \mu \mathrm{M} \mathrm{CuSO}$ for 3 days and then transferred to the media containing 1 or $50 \mu \mathrm{M} \mathrm{CuSO}_{4}$ for 7 days. Three independent lines for each construct were shown. (c,d) $\mathrm{Cu}$ concentration in the shoots and roots of OsHMA4 transgenic lines grown on the media containing $1 \mu \mathrm{M}(\mathbf{c})$ or $50 \mu \mathrm{M} \mathrm{CuSO}_{4}(\mathbf{d})$. Plants were grown as in (a) and the same lines as in $\mathbf{b}$ were used. Cu concentration was determined by ICP-MS. Data in b-d were shown as means \pm s.d. with $n=9$ to 16 in $\mathbf{b}$ and $n=3$ in $\mathbf{c}$ and $\mathbf{d}$. Columns with different letters in $\mathbf{b}$-d indicate significant difference ( $P \leq 0.01$, Fisher's LSD test). DW, dry weight.

ZIP2, which was induced in shoots of some lines (Supplementary Fig. 9d).

To characterize the $\mathrm{Cu}$ transport activity of OsHMA4, we expressed OsHMA4 in the yeast WT strain BY4741 using a low copy number centromeric plasmid pYEC2/CT-GFP. GFP signals were specifically observed at the vacuolar membrane of yeast transformed with either pYEC2-OsHMA4(TQ)-GFP or pYEC2-OsHMA4(LM)-GFP (Fig. 5a), supporting the tonoplast localization of OsHMA4 we observed in both rice and Arabidopsis. Yeast expressing OsHMA4-GFP were more tolerant 

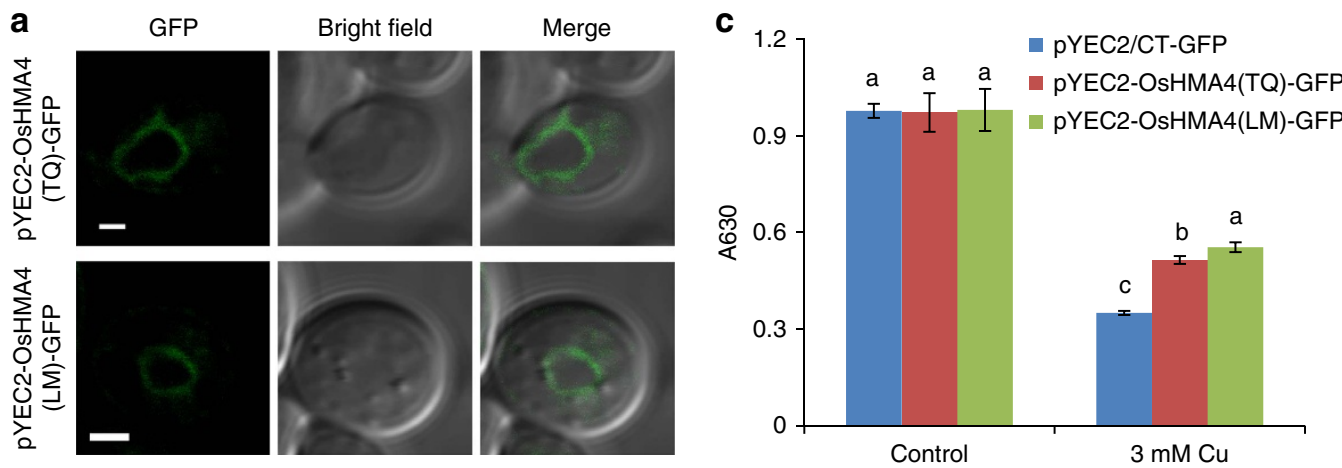

b
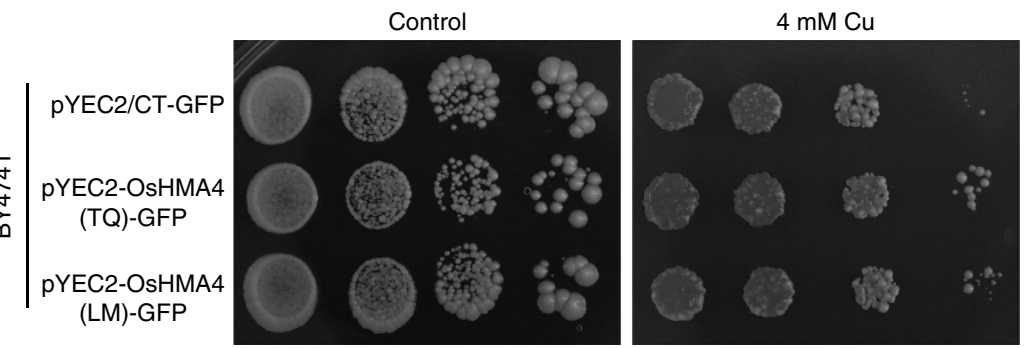

Figure 5 | Functional analysis of OsHMA4 in yeast. (a) Subcellular localization of OsHMA4 in yeast. OsHMA4 from TeQing (OsHMA4(TQ)) or from Lemont (OsHMA4(LM)) were expressed in a centromeric plasmid pYEC2/CT-GFP. GFP signals were observed mainly on the tonoplast. Bar, $1 \mu \mathrm{m}$. (b) Expression of OsHMA4 in yeast using a centrimeric plasmid pYEC2/CT-GFP enhances Cu tolerance. Overnight yeast cell suspension of BY4741 transformed with empty vector PYEC2/CT-GFP or OsHMA4 from TQ or LM were serially diluted (1:10) and spotted on the media without (Control) or with $4 \mathrm{mM} \mathrm{CuSO}_{4}$. Pictures were taken after 5 days growth at $30^{\circ} \mathrm{C}$. (c) The absorbance at $630 \mathrm{~nm}$ (A630) of cell cultures of BY4741 transformed with the empty vector pYEC2/CT-GFP or OsHMA4-GFP from TQ or LM. Yeast strains were grown in liquid media containing without (Control) or with $3 \mathrm{mM}$ CuSO 4 for $24 \mathrm{~h}$. Data were shown as means \pm s.d. with three independent colonies. Columns with different italic or non-italic letters indicate significant difference under control or $3 \mathrm{mM} \mathrm{CuSO}_{4}$, respectively ( $P \leq 0.01$, Fisher's LSD test).

to $\mathrm{Cu}$ stress than those transformed with the empty vector (Fig. 5c,d), supporting a function for OsHMA4 in transport of $\mathrm{Cu}$ into yeast vacuoles. Expression of the cucumber tonoplastlocalized CsHMA5.1 and CsHMA5.2 using a similar low copy number centromeric plasmid has also been shown to improve $\mathrm{Cu}$ tolerance in yeast ${ }^{26}$.

To further test the $\mathrm{Cu}$ transport activity of OsHMA4, we expressed OsHMA4 in the yeast $c c c 2$ mutant using a high copy number $2 \mu$ origin plasmid pYES2. The Cu-transporting P-type ATPase CCC2 localizes at the late- or post-Golgi compartment and delivers $\mathrm{Cu}$ to the multi-copper oxidase Fet3P, which is required for high-affinity $\mathrm{Fe}$ uptake at the plasma membrane $\mathrm{e}^{27}$. The ccc2 knockout mutant is unable to grow on Fe-deficient media (Supplementary Fig. 12a). We observed that expression of OsHMA4 from either TQ or LM in the $c c c 2$ yeast mutant was able to restore growth of the yeast mutant on Fe-deficient media, suggesting that OsHMA4 has $\mathrm{Cu}$ transport activity in yeast (Supplementary Fig. 12a). Analysis of the GFP signal in yeast expressing OsHMA4-GFP from the high copy number $2 \mu$ origin pYES2 plasmid revealed that OsHMA4-GFP localizes throughout the endomembrane system, likely including the late- or post-Golgi compartment (Supplementary Fig. 12f). Such mislocalization of OsHMA4-GFP to the late- or post-Golgi compartment when accumulated to high levels explains how OsHMA4 can complement the $c c c 2$ growth defect when expressed from pYES2. Supporting this conclusion that OsHMA4 can transport $\mathrm{Cu}$ in yeast, we also observed that expressing OsHMA4 in either WT yeast or a yeast strain lacking the high-affinity $\mathrm{Cu}$ transporter CTR1 increased their sensitivity to excess $\mathrm{Cu}$ (Supplementary Fig. 13a). Furthermore, expression of OsHMA4 did not alter the sensitivity of yeast to other heavy metals such as $\mathrm{Cd}, \mathrm{Co}, \mathrm{Ag}, \mathrm{Pb}, \mathrm{Mn}$ and $\mathrm{Zn}$ (Supplementary Fig. 13b), suggesting that OsHMA4 transports Cu specifically. Significantly, WT yeast expressing the LM OsHMA4 allele was more sensitive to excess $\mathrm{Cu}$ than WT yeast expressing the TQ OsHMA4 allele (Supplementary Fig. 12b-e), suggesting stronger $\mathrm{Cu}$ transport activity of OsHMA4 from LM. The increased sensitivity to $\mathrm{Cu}$ of yeast expressing OsHMA4 from pYES2 can again be explained by the mislocalization of OsHMA4 to internal membrane compartments that are sensitive to enhanced $\mathrm{Cu}$ transport. This is similar to previous studies in which expression of the normally tonoplast-localized OsHMA3 or AtHMA3 in yeast using the high copy number plasmid pYES2 increases the sensitivity to Cd stress rather than enhancing Cd tolerance ${ }^{28}$.

Analysis of genetic variation of OsHMA4. To gain insight into the contribution of genetic variation at OsHMA4 to variation in grain $\mathrm{Cu}$ across the $O$. sativa species, we analysed the sequence of OsHMA4 in the genomes of 950 diverse worldwide rice accessions $^{29}$. This analysis revealed nine non-synonymous polymorphisms in the coding sequence of OsHMA4, including the polymorphic V914A we discovered between TQ and LM (Supplementary Table 2; Supplementary Fig. 2; Fig. 1e). The allele frequency of the strong allele of OsHMA4 from LM (A914) was very low (0.0068), suggesting that it is a rare allele (Supplementary Table 2). To further associate genetic variation at OsHMA4 with rice grain $\mathrm{Cu}$, we genotyped OsHMA4 in 1,349 worldwide accessions from the USDA Rice Core Collection for which we had previously reported the concentration of grain $\mathrm{Cu}^{30}$. We identified five of the previous nine non-synonymous polymorphisms and also identified 67 accessions with the LM-like strong allele (5.1\%; Supplementary Table 2). The accessions with the strong OsHMA4 allele (A914) generally accumulate less grain $\mathrm{Cu}$ and are largely distributed in the USA (26 of 67; Fig. 6a,b). When taking the kinship between the accessions into account, we only observed a significant difference in grain $\mathrm{Cu}$ between the two 
a

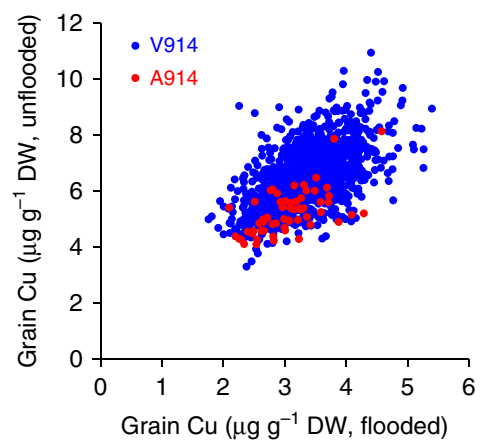

C

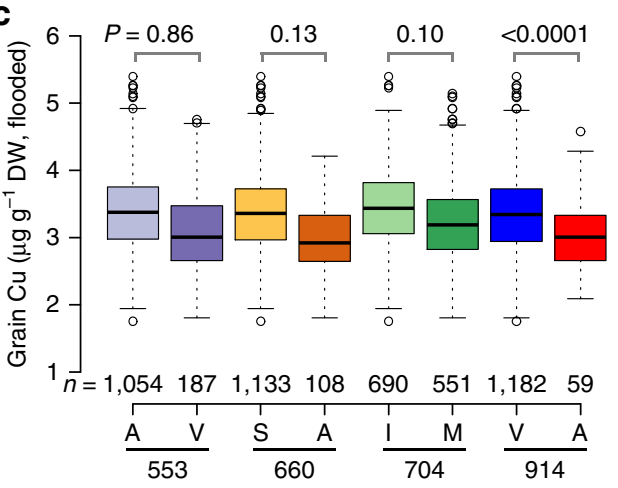

b

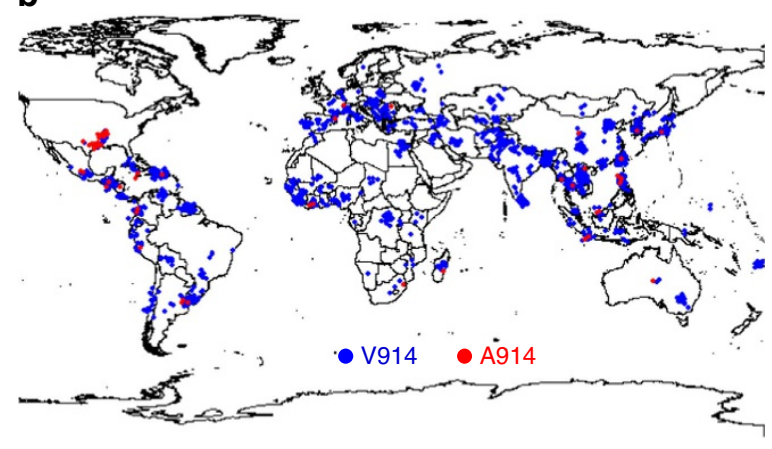

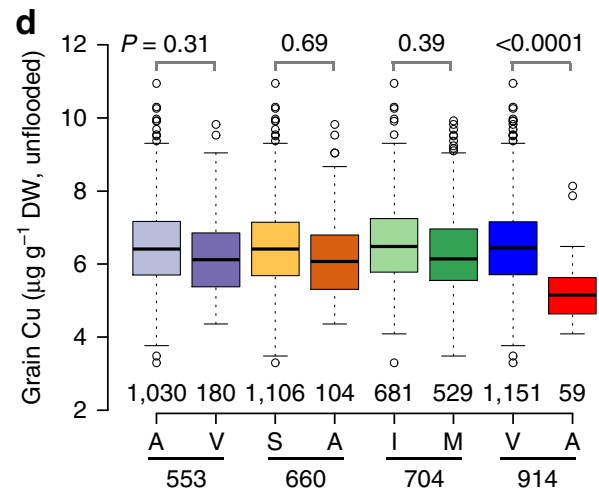

Figure 6 | Natural allelic variation at the OsHMA4 loci underlies the variation of grain $\mathbf{C u}$ in rice. (a) The grain Cu of USDA core collection rice accessions with the strong (A914) and weak allele (V914) of OsHMA4 when grown under flooded or unflooded condition. (b) Distribution of rice accessions with the strong and weak allele of OsHMA4. (c,d) The grain $\mathrm{Cu}$ of rice accessions with different OsHMA4 haplotypes grown under flooded (c) or unflooded condition (d). Data are shown as boxplots. Numbers under the boxes are the accession number; numbers above the boxes are the $P$ values generated by the generalized least squares model. DW, dry weight. The map image was generated using R package rworldmap ${ }^{64}$ with data derived from Natural Earth v1.4.0 (http://www.naturalearthdata.com/).

alleles polymorphic at the V914A site, but not at the other three polymorphic sites that have a minor allele frequency higher than 0.05 (Fig. 6c,d). These polymorphisms explain a significant amount of the variation in grain $\mathrm{Cu}$ of this diverse core collection when grown in either flooded $\left(8.3 \% ; \mathrm{F}_{4}, 1,236=28, P<0.0001\right.$, generalized least squares approach, $n=1,182 / 59)$ or unflooded conditions $\left(8.6 \% ; \mathrm{F}_{4,1,205}=28.55, P<0.0001\right.$, generalized least squares approach, $n=1,151 / 59)$. One likely source of the strong allele we first identified in LM is its ancestor Fortuna, which was selected from a landrace from Taiwan, suggesting that the strong allele may originate from Asia (Supplementary Fig.14a). This strong allele of OsHMA4 was not found in 446 accessions of wild rice Oryza rufipogon, the immediate ancestral progenitor of Asian cultivated rice Oryza sativa, or in African rice Oryza glaberrima (20 accessions) and its progenitor Oryza barthii (94 accessions) $^{31,32}$. Grain $\mathrm{Cu}$ is generally lower when plants are grown in flooded compared with unflooded paddy conditions (Fig. 6c,d), which is likely due to lower $\mathrm{Cu}$ bioavailability in water-logged soil. The reasons for this reduction in $\mathrm{Cu}$ bioavailability under flooded conditions are complex and involve a decrease in redox potential ${ }^{33}$.

\section{Discussion}

To cope with the dual nature of $\mathrm{Cu}$ being essential for cells and toxic when present in excess, plants have evolved sophisticated mechanisms to control the cellular $\mathrm{Cu}$ concentration. One strategy is to sequester excess $\mathrm{Cu}$ into vacuoles. This vacuolar $\mathrm{Cu}$ serves as a reservoir for $\mathrm{Cu}$ that can be remobilized under $\mathrm{Cu}$ deficiency. However, the molecular mechanism underlying this process in plants is poorly understood. In this study, we identified a QTL controlling rice grain $\mathrm{Cu}$ and determined the likely causal gene to be OsHMA4. OsHMA4 belongs to the $\mathrm{Cu}^{+} / \mathrm{Ag}^{+}$ subgroup of HMA and we provide evidence it encodes a tonoplast-localized transporter specific for $\mathrm{Cu}$. We found that the genotypic difference in grain $\mathrm{Cu}$ was not due to the expression level or subcellular localization of OsHMA4, but most likely the transport activity for $\mathrm{Cu}$ (Supplementary Fig. 1b; Fig. 3g; Supplementary Fig. 8e; Supplementary Fig. 12b-e). OsHMA4 from the low $\mathrm{Cu}$ cultivar (LM) showed stronger transport activity for $\mathrm{Cu}$ than that from the high $\mathrm{Cu}$ cultivar (TQ) when expressed in both Arabidopsis and yeast (Figs 4a,b and 5c; Supplementary Fig. 12b-e). Knockout of OsHMA4 resulted in increased $\mathrm{Cu}$ concentration in the shoots and xylem sap, but decreased $\mathrm{Cu}$ in the roots and root cell sap (Fig. 2e-h). We conclude that OsHMA4 most likely functions to sequester $\mathrm{Cu}$ into vacuoles of pericycle cells to help control the root-to-shoot translocation of $\mathrm{Cu}$. This likely vacuolar sequestration of $\mathrm{Cu}$ by OsHMA4 is also associated with enhanced $\mathrm{Cu}$ tolerance (Fig. 2i,j; Supplementary Fig. 5i).

Several transporters have been shown to compartmentalize various heavy metals in root vacuoles. For example, Arabidopsis AtHMA3 participates in vacuolar sequestration of $\mathrm{Cd}, \mathrm{Zn}, \mathrm{Co}$ and $\mathrm{Pb}^{34,35}$, and AtMTP3 is involved in sequestration of $\mathrm{Zn}^{36}$. Recently, two cucumber $\mathrm{P}_{1 \mathrm{~B}}$-type ATPases CsHMA5.1 and CsHMA5.2 have been shown to transport $\mathrm{Cu}$ into vacuoles in yeast ${ }^{26}$. However, their in vivo functions in regulating $\mathrm{Cu}$ homoeostasis remains unclear. In Arabidopsis, the protein mediating the transport of $\mathrm{Cu}$ into vacuoles has not been identified. OsHMA5 from rice and AtHMA5 from Arabidopsis are involved in loading $\mathrm{Cu}$ into the xylem for root-to-shoot 
translocation and/or $\mathrm{Cu}$ detoxification in roots ${ }^{12,13,18}$. Several other HMA proteins in Arabidopsis have been shown to have $\mathrm{Cu}$-transporting activity but none of them are involved in the efflux of $\mathrm{Cu}$ into vacuoles. For example, AtHMA6/PAA1 and AtHMA8/PAA2 are required for $\mathrm{Cu}$ transport in chloroplasts and AtHMA7/RAN1 delivers $\mathrm{Cu}$ to ethylene receptors on the Golgi membrane ${ }^{14-17}$. Even though the functional counterpart of OsHMA4 is likely absent in Arabidopsis, several OsHMA4 homologues have been identified in other species such as sorghum, poplar and grape vine ${ }^{26}$.

Expression of OsHMA4 was not induced by short-term $\mathrm{Cu}$ treatment in a previous study ${ }^{37}$. However, here we demonstrated that OsHMA4 is induced under longer-term exposure to excess $\mathrm{Cu}$ (Fig. 3b,c). This is consistent with the proposed function of OsHMA4 in compartmentalization of $\mathrm{Cu}$ into vacuoles after exposure to excess $\mathrm{Cu}$. Furthermore, the expression of OsHMA4 is suppressed by $\mathrm{Cu}$ deficiency (Fig. 3d). Such downregulation under $\mathrm{Cu}$ deficiency may decrease $\mathrm{Cu}$ sequestration in the vacuolar storage pool.

OsHMA4 is characteristically localized at the pericyle cells of root mature zones (Fig. 3e). This localization is similar to OsHMA5, a plasma membrane-localized $\mathrm{Cu}$ transporter responsible for xylem loading of $\mathrm{Cu}^{18}$. This suggests that OsHMA4 plays a role in fine tuning cellular $\mathrm{Cu}$ concentration before loading to the xylem depending on $\mathrm{Cu}$ concentration in the environment. This localization is also different from other tonoplast-localized transporter in rice roots such as OsHMA3, which is localized in all root cells ${ }^{28}$. Furthermore, OsHMA4 is also characterized by its transport specificity for $\mathrm{Cu}$ (Supplementary Fig. 6a-e; Supplementary Fig. 13b). Among the HMA members characterized in rice, OsHMA2 and OsHMA3 transport both $\mathrm{Cd}$ and $\mathrm{Zn}^{38-41}$, while OsHMA9 transports $\mathrm{Cu}$, $\mathrm{Zn}, \mathrm{Pb}$ and $\mathrm{Cd}^{37}$. The mechanisms underlying the transport substrate specificity remain to be examined.

The strong allele of OsHMA4 from LM results from a single amino acid change at position 914 (from V to A; Fig. 1d). This amino acid is localized in the seventh transmembrane domain (Fig. 1e,f; Supplementary Fig. 2), which is predicated to be the Cu-binding site I including the conserved YN and CPC motifs 22 (Fig. 1e,f; Supplementary Fig. 2). Thus, the V914A variant might differ in $\mathrm{Cu}$ binding activity and thus have different transporting activity. The absence of the strong allele of OsHMA4 (A914) in wild rice $O$. rufipogon, the immediate ancestral progenitor of Asian cultivated rice $O$. sativa, suggested that the weak OsHMA4 allele (V914) is ancestral, and the strong allele might have arisen during the domestication of $O$. sativa ${ }^{29}$. However, the lack of evidence for a selective sweep of the genome around OsHMA4 suggests that this strong allele of OsHMA4 was not selected during domestication ${ }^{29}$. The strong allele of OsHMA4 is rare in the worldwide rice population perhaps explaining why this functional polymorphism was not identified in a recent genome-wide association study ${ }^{42}$.

In summary, we have identified OsHMA4 as the most likely causal gene underlying the QTL for $\mathrm{Cu}$ accumulation in rice grain through multiple year field and greenhouse trials. OsHMA4 localizes to the vacuolar membrane and we provide evidence that it functions to transport $\mathrm{Cu}$ into vacuoles in roots. The identification of natural variation at the OsHMA4 loci associated with rice grain $\mathrm{Cu}$ provides an efficient way to breed rice varieties with $\mathrm{Cu}$ enrichment in the grain, which may be helpful in solving global $\mathrm{Cu}$ micronutrient deficiency. The novel OsHMA4 alleles identified and the molecular markers developed in this work can be directly used in breeding to develop rice varieties with grain $\mathrm{Cu}$ concentrations that are tuned to both the concentration of $\mathrm{Cu}$ in the soil and the dietary needs of the population's consuming the grain.

\section{Methods}

Plant materials and growth conditions. The LT-RILs were derived from a cross between a US tropical japonica rice cultivar Lemont (LM) and a Chinese indica cultivar TeQing (TQ) by single-seed descent ${ }^{43,44}$. The LT-RILs contained 280 lines and were genotyped using 175 restriction fragment length polymorphism markers ${ }^{44}$. The LT-RILs grown in the field in 2002, 2003, 2006, 2007 and 2008 were in the $\mathrm{F}_{15}, \mathrm{~F}_{16}, \mathrm{~F}_{17}, \mathrm{~F}_{18}$ and $\mathrm{F}_{19}$ generations, respectively. The TILs contain 123 lines and were originally genotyped using 168 simple sequence repeat (SSR) markers ${ }^{45}$. Plants were grown under flooded and unflooded condition in 2007 and 2008 as described previously ${ }^{20}$. Due to a storm, the LT-RILs grown under unflooded condition were destroyed in 2007 and thus were not included in the analysis. In 2010, the TIL population was grown in a greenhouse at Purdue University, West Lafayette, Indiana, USA. Plants were grown in $10 \times 10 \times 12 \mathrm{~cm}$ pots with sandy soil Profile Greens Grade (Profile Products, LLC, Illinois, USA) and the irrigation was controlled by an automatic system. Plants were irrigated every day with tap water to maintain the water level to $1 / 3$ of the pot depth, and fertilized once a week with water-soluble fertilizer (15N-1.3P-13.3K; Greencare Fertilizers, Kankakee, Illinois, USA) until seeds were collected.

HIFs were generated as previously described ${ }^{23,24}$. A derived cleaved amplified polymorphic sequences (dCAPS) marker was developed based on the causal polymorphism of T4656C on OsHMA4 between TQ and LM. TIL626 was identified as heterozygous on this dCAPS. The plants fixed with TQ allele (HIF626-TQ) and plants fixed with LM allele (HIF626-LM) were selected in self-pollinated progeny plants of TIL626.

The T-DNA insertion mutant for OsHMA4 (PFG_1B-07418) was obtained from Kyung Hee University, Korea (http://cbi.khu.ac.kr/RISD_DB.html) ${ }^{46}$. The progeny plants segregated from 1B-07418 without the T-DNA insertion in the OsHMA4 gene were used as a WT control. The mutant and WT were genotyped by PCR and expression level of OsHMA4 in the mutant was determined by reverse transcriptase-PCR (RT-PCR; Supplementary Fig. 4b,c; see full gel images in Supplementary Fig. 15a). For analysis of the grains and different tissues of the WT and the oshma 4 mutant, plants were grown in the greenhouse. For the hydroponic experiment, WT and the oshma4 mutant were grown as previously described ${ }^{47}$ Seeds of WT and oshma4 were kept at $42^{\circ} \mathrm{C}$ for at least 1 week to break any possible dormancy, soaked in water at room temperature for 2 days, and then germinated at $37^{\circ} \mathrm{C}$ for 1 day. The most uniformly germinated seeds were sown in a 96-well plate from which the bottom was removed. The plate was put in a pipette tip box $(12 \times 8.5 \times 7.5 \mathrm{~cm})$ and floated in water for 1 days at $37^{\circ} \mathrm{C}$ in the dark to encourage root growth. Plants were propagated in a growth chamber with a $12-\mathrm{h}$ light $\left(26^{\circ} \mathrm{C}\right) / 12$-h dark $\left(22^{\circ} \mathrm{C}\right)$ photoperiod, $50-60 \%$ relative humidity and $700 \mu \mathrm{mol} \mathrm{m}{ }^{-2} \mathrm{~s}^{-1}$ light. After 5 days, the seedlings were cultured with halfstrength Kimura B solution ${ }^{18}$ and the nutrient solution was renewed every 2 days. The nutrient solution was prepared using deionized water except for the element deficiency experiment in which Milli-Q water was used. For some experiments, the WT (cv. Dongjin) and T-DNA mutant were grown hydroponically in a closed greenhouse of the Institute of Plant Science and Resources, Okayama University as described previously ${ }^{18}$.

Tissue elemental analysis. The elemental concentration of the grain and other organs was determined using an inductively couple plasma mass spectrometer (ICP-MS; Elan DRCe, PerkinElmer; or NexION 300D, PerkinElmer) as described previously ${ }^{20}$. The grains of LT-RILs and TILs were dehulled using a modified Satake TH035A sheller (Satake Engineering, Co. Ltd., Tokyo, Japan) with the rubber liner on the rollers replaced with PU40 Polyurethane plastic to prevent the contamination of $\mathrm{Zn}^{20}$. Grains of WT and the oshma4 mutant were dehulled manually. For determination of elemental concentrations in the blade, sheath, rachis, node and internode, tissues were washed with Milli-Q water and dried at $88^{\circ} \mathrm{C}$ overnight. For analysis of roots from hydroponically grown plants, roots were excised from the plants, washed with $0.5 \mathrm{mM} \mathrm{CaCl}_{2}$ solution three times, rinsed with Milli-Q water once and dried at $88^{\circ} \mathrm{C}$ overnight. Samples were digested with concentrated $\mathrm{HNO}_{3}$ at $118^{\circ} \mathrm{C}$ for $4 \mathrm{~h}$ before ICP-MS analysis.

QTL analysis and fine mapping of $\mathbf{q G C u 2}$. QTL analyses were performed using Windows QTL cartographer version 2.5 (http://statgen.ncsu.edu/qtlcart/ WQTLCart.htm) using composite interval mapping. The composite interval mapping analysis was run using Model 6 with forward and backward stepwise regression, a window size of $10 \mathrm{cM}$, and a walk speed of $1 \mathrm{cM}$. The threshold for detection of a QTL was set at a LOD score of 3.0 following 1,000 permutations at 0.05 significant level. For fine mapping of $q G C u 2-1$, four LT-RILs (LT:390, LT:528 LT:550, LT:597) with the TQ genotype at the mapping region were crossed with LM. $F_{2}$ population was generated by self-pollination $F_{1}$ s. Two markers $\mathrm{H} 24454$ and $\mathrm{H} 26652$ were used to detect the recombinantion events that occurred around $q G C u 2-1$ and 12 molecular markers were developed for fine mapping. Five recombinants were isolated from $1,258 \mathrm{~F}_{2}$ plants and fixed recombinant $\mathrm{F}_{3}$ plants were generated by self-pollination. The $\mathrm{Cu}$ concentration in the grain and leaf of fixed recombinants was determined by ICP-MS. By progeny testing, qGCu2-1 was fine mapped between the markers RM3294 and RM6378.

Transgenic complementation test. For transgenic complementation experiment oshma4 was transformed with OsHMA4 under the control of its own promoter. 
The coding region of OsHMA4 linked with the nopaline synthase (NOS) terminator was amplified using the plasmid GFP-OsHMA4 (described below) as the template. The fragment was digested with BamHI and BglII and then subcloned into the binary vector pTF101.1 (ref. 48), which was digested with BamHI and dephosphorylated with Shrimp Alkaline Phosphatase (Takara). The 3,016 bp region upstream of the initiation codon of OsHMA4 was amplified by PCR from the genomic DNA. The fragment was digested with SpeI and BamHI and then ligated into the XbaI-BamHI site of pTF101.1-OsHMA4. After being sequenced for confirmation (ABI PRISM 3,130 Genetic Analyzer, Applied Biosciences), the resulting plasmid pOsHMA4:OsHMA4 was subsequently introduced into Agrobacterium tumefaciens (strain EHA101). Callus was induced from mature embryos of the oshma4 mutant for Agrobacterium-mediated transformation ${ }^{49}$. The expression level of OsHMA4 in transgenic plants was detected by RT-PCR. For grain $\mathrm{Cu}$ determination, the complementation lines, WT rice and the oshma4 mutant were transplanted into 3.51 plastic pots filled with paddy soil. The plants were grown in a closed greenhouse under natural light at $25-30^{\circ} \mathrm{C}$ until mature. Brown rice was collected for digestion and measurement as described above. The primer sequences used are listed in Supplementary Table 3.

Tissue specificity of OsHMA4 expression. To investigate the tissue and cellular specificity of OsHMA4 expression, the GFP-OsHMA4 fusion was expressed in the background of the oshma4 mutant under the control of its own promoter $(3,016 \mathrm{bp})$. The full-length complementary DNA (cDNA) of OsHMA4 was ligated to the $3^{\prime}$ end of GFP with the coding sequence for seven additional amino acids (SGGGGGG) digested with BspEI to generate the plasmid GFP-OsHMA4 (ref. 37). The fused GFP-OsHMA4 fragment was inserted between the promoter fragment and NOS terminator to produce the pOsHMA4:GFP-OsHMA4 plasmid. This construct was introduced into the oshma4 T-DNA knockout mutant as described above.

For further analysis of the tissue expression pattern of OsHMA4, the 2,630-bp promoter sequence of OsHMA4 was PCR amplified and subcloned into the PstI-BamHI site of pTF101.1-GUS vector (modified from pTF101.1 (ref. 48) by insertion of the sequence of GUS into the HindIII-EcoRI site of pTF101.1). The resulting plasmids were transformed into rice japonica cv. Nipponbare. Rice transformation was performed in Iowa State University Plant Transformation Facility. GUS histochemical staining was performed as described previously ${ }^{46}$. The primer sequences are listed in Supplementary Table 3.

\section{Subcellular localization and tissue expression pattern of OsHMA4.}

To investigate the subcellular location of OsHMA4, the full-length coding sequence of OsHMA4 was amplified from cDNA synthesized from TQ or LM using the primers listed in Supplementary Table 3, and ligated into the XbaI-BamHI site of p1301GFP vector ${ }^{4}$. The resulting plasmids 35S:GFP-OsHMA4(TQ) and 35S:GFP-OsHMA4(LM) were transformed into A. tumeraciens strain GV3101 and introduced into the Arabidopsis AtHMA5 mutant (SALK_040252) using the floral dip method ${ }^{50}$. The roots of T3 transgenic plants were examined using a confocal laser scanning microscope (Carl Zeiss LSM700). GFP was excited using an argon laser at $488 \mathrm{~nm}$ and the emission was collected between 505 and $530 \mathrm{~nm}$. To visualize the nuclei, roots were incubated with $1 \mu \mathrm{g} \mathrm{ml}^{-1}$ of 4,6-diamidino-2phenylindole (Molecular Probes) for $5 \mathrm{~min}$ at room temperature and washed five times with PBS buffer. 4,6-diamidino-2-phenylindole were excited with a ultraviolet laser at $395 \mathrm{~nm}$.

Immunostaining and western blot analysis. Immunostaining was carried out on roots of WT and transgenic rice plants expressing GFP-OsHMA4 driven by OsHMA4 native promoter in oshma4 using an antibody against GFP (A-11122; Molecular Probes) as described previously ${ }^{18}$. For western blot analysis, the WT and transgenic lines expressed expressing GFP-OsHMA4 driven by OsHMA4 native promoter in oshma4 were used. Plants were grown hydroponically for 35 days in the half-strength Kimura B solution, and then treated with $2 \mu \mathrm{M} \mathrm{Cu}$ for $6 \mathrm{~h}$ before harvesting for protein extraction. The microsome isolation and fractionation were performed according to the method described previously with slight modifications ${ }^{28}$. The suspended microsomes were fractionated with discontinuous sucrose gradients $(20-60 \%$ sucrose in $10 \mathrm{mM}$ Tris- $\mathrm{HCl}$, pH 7.6, $1 \mathrm{mM}$ EDTA, and $1 \mathrm{mM}$ DTT) by ultracentrifugation at $100,000 \mathrm{~g}$ for $18 \mathrm{~h}$. The fractionated membranes were recovered by ultracentrifugation at $100,000 \mathrm{~g}$ for $40 \mathrm{~min}$. Each pellet was resuspended for the concentration measurement and further analysis. Equal amounts of samples were incubated at $95^{\circ} \mathrm{C}$ for $2 \mathrm{~min}$ and then loaded into the SDS-PAGE gels (5-20\% gradient polyacrylamide, ATTO, Japan). The transfer to polyvinylidene difluoride membrane was performed with a semidry blotting system, and the membrane was treated with the purified primary rabbit anti-GFP (A-11122; Molecular Probes; 20,000 times dilution) in an Immnunoreaction Enhancer Solution (Can Get Signal, TOYOBO, Japan), anti-V-ATPase (AS07213, Agrisera; 10,000 times dilution), anti- $\mathrm{H}^{+}$-ATPase polyclonal antibodies (AS07260, Agrisera; 10,000 times dilution), and Anti-Bip (COP-080017, Cosmo bio; 10,000 times dilution). ECL peroxidase-labelled anti-rabbit antibody (W4011, Promega; 10,000 times dilution) was used as a secondary antibody, and an ECL Plus western blotting detection system (GE Healthcare) was used for detection via chemiluminescence (Bio-Rad). The protein amount was $30 \mu \mathrm{g}$ for microsome,
$5 \mu \mathrm{g}$ for the GFP detection, $1 \mu \mathrm{g}$ for V-ATPase, $\mathrm{H}^{+}$-ATPase and Bip detection. Full images of western blot were shown in Supplementary Fig. 15b.

Xylem sap and root cell sap analysis. Collection of xylem sap was performed as previously described with modifications ${ }^{51}$. WT and oshma4 plants were hydroponically grown in a growth chamber for 5 weeks in half-strength Kimura B solution with $\mathrm{Cu}$ omitted. The plants were then transferred to a nutrient solution containing either 0.2 or $2 \mu \mathrm{M}$ added $\mathrm{CuSO}_{4}$. After 1 week of treatment, the shoots were cut with a razor at about $2 \mathrm{~cm}$ above the root-shoot junction. The xylem sap was collected for $1 \mathrm{~h}$ after cutting. The first drop of xylem sap emerging was discarded to prevent contamination of the contents from damaged cells. Xylem sap from 16 plants was combined as one replicate and three replicates were made. The $\mathrm{Cu}$ concentration in the xylem sap was determined by ICP-MS. Root cell sap was prepared according to Ueno et al. ${ }^{28}$ with modifications. Briefly, WT and oshma4 mutant plants were grown hydroponically in half-strength Kimura B nutrient solution for 2 weeks and transferred to nutrient solution containing either 0.2 or $2 \mu \mathrm{M} \mathrm{CuSO}_{4}$ for 7 days. Root cell sap was prepared from the root tips as follows. After washing entire root systems three times with $0.5 \mathrm{mM} \mathrm{CaCl}_{2}$, the first $1-2 \mathrm{~cm}$ of root tip segments were cut, rinsed with Milli-Q water and blotted dry with tissue paper. One root segment from each of 16 plants comprised a single sample, and a total of 4 samples were run per $\mathrm{Cu}$ growth treatment. Each sample of 16 root segments were put in a $0.22 \mu \mathrm{m}$ filter unit (Ultrafreer-MC; Millipore) and centrifuged at $3,000 \mathrm{~g}$ for $10 \mathrm{~min}$ at $4{ }^{\circ} \mathrm{C}$ to remove the apoplastic solution. Root segments were then frozen at $-80^{\circ} \mathrm{C}$ overnight. After thawing at room temperature for a short time, samples were centrifuged at $20,600 \mathrm{~g}$ for $10 \mathrm{~min}$ to collect the root cell sap solution. Five microliter of each root cell sap sample were digested with $1 \mathrm{ml}$ concentrated $\mathrm{HNO}_{3}$ at $118^{\circ} \mathrm{C}$ for $1 \mathrm{~h}$ and the $\mathrm{Cu}$ concentration was determined using ICP-MS

Cu tolerance evaluation. To compare the $\mathrm{Cu}$ tolerance of WT and the oshma4 mutant, seeds of each were soaked in tap water for 2 days at $30^{\circ} \mathrm{C}$ and then transferred to a net floating on a $0.5 \mathrm{mM} \mathrm{CaCl}_{2}$ solution for 3 days. At day 4 , seedlings were exposed to a $0.5 \mathrm{mM} \mathrm{CaCl}_{2}$ solution ( $\mathrm{pH}$ 5.6) containing either no added $\mathrm{CuSO}_{4}$ or $\mathrm{CuSO}_{4}$ added at 100, 200, 400, $600 \mathrm{nM}$ for $24 \mathrm{~h}$. The experiment was performed at $25^{\circ} \mathrm{C}$. The root length of each seedling was measured before and after the treatments, and relative root elongation (=(root elongation with $\mathrm{Cu}) /$ (root elongation without $\mathrm{Cu}) \times 100$ ) was calculated. Twelve seedlings for each treatment were used. A long-term treatment with excess $\mathrm{Cu}$ was also performed by exposing WT, the oshma4 mutant and two independent transgenic complementation lines to a nutrient solution with or without $2 \mu \mathrm{M}$ added $\mathrm{CuSO}_{4}$ for 15 days at $25^{\circ} \mathrm{C}$. Shoot length was recorded and the normalized increased plant height was calculated as (plant height increase with $\mathrm{Cu}$ )/(plant height increase without $\mathrm{Cu}) \times 100$.

Quantitative real-time PCR. To investigate the expression pattern of OsHMA4 at different growth stages, different tissues from plants (cv Nipponbare) grown in a paddy field were harvested for RNA extraction and cDNA preparation ${ }^{18}$. The tissue-specific expression of OsHMA4 in roots was examined with the help of laser microdissection according to the described methods ${ }^{18}$. The relative expression of OsHMA4 was investigated by quantitative real-time RT-PCR using the HistoneH3 gene as the internal control. To determine the expression of OsHMA4 in response to different metals in the nutrient solution, total RNA was extracted from shoots and roots using a TRIzol Plus RNA Purification kit (Invitrogen, Life Technologies), and then treated with a PureLink DNase Set (Invitrogen, Life Technologies) to remove potential genomic DNA contamination. The cDNA synthesis was carried out using a SuperScript VILO cDNA Synthesis Kit (Invitrogen, Life Technologies). Quantitative real-time PCR was performed on an ABI StepOnePlus Real-Time PCR System (Applied Biosystems) with Maxima SYBR Green qPCR Master Mixes (Thermo Scientific). Ct values were normalized to the corresponding endogenous control gene (LOC_Os03g50885). The $\Delta \Delta \mathrm{Ct}$ method was used for quantitative RT-PCR analysis. The primer sequences are listed in Supplementary Table 3. Total RNA was extracted from yeast cells using a PureLink RNA Mini Kit (Thermo Fisher Scientific). The cDNA synthesis and quantitative real-time PCR was performed was performed as above. The yeast housekeeping gene AGL9 was used as an endogenous control gene.

Expressing OsHMA4 in Arabidopsis AtHMA5 mutant and WT Col-0. The construction of the 35S:GFP-OsHMA4(TQ) and 35S:GFP-OsHMA4(LM) vectors was described above. To generate the AtHMA5 promoter driven OsHMA4 expression vectors, the 2,900-bp promoter sequence of AtHMA5 was PCR amplified from the genomic DNA of Col-0 and ligated into the PstI-SalI site of the pCambial301 vector. The fragments containing OsHMA4 coding sequence fused in-frame to GFP were released from the 35S:GFP-OsHMA4(TQ) or 35S:GFP-OsHMA4(LM) vectors and inserted into the SalI-EcoRI site of above vector ( $p$ Cambia1301-AtHMA5pro) to generate the AtHMA5pro:GFP-OsHMA4(TQ) and AtHMA5pro:GFP-OsHMA4(LM) vectors. The resulting plasmids were transformed into A. tumeraciens strain GV3101. The 35S:GFP-OsHMA4(TQ) or 35S:GFP-OsHMA4(LM) were introduced into the Arabidopsis athma5 mutant (SALK_040252) or Col-0 and AtHMA5pro:GFP-OsHMA4(TQ) and 
AtHMA5pro:GFP-OsHMA4(LM) were introduced into the athma5 mutant as described above. For the $\mathrm{Cu}$ tolerance assay, $\mathrm{T} 3$ transgenic plants were grown on MGRL medium containing $50 \mathrm{mg} \mathrm{ml}^{-1}$ hygromycin for 3 days and the positive plants were transferred to hygromycin-free MGRL medium containing 1 or $50 \mu \mathrm{M}$ added $\mathrm{CuSO}_{4}$ for 7 days. To quantify tolerance to $\mathrm{Cu}$ in the growth medium root length was measured by marking the position of root tips on the Petri dish at the indicated times.

Functional analysis of OsHMA4 in yeast. The yeast (Saccharomyces cerevisiae) WT strain BY4741 (MATa his $3 \Delta 1$ leu2 $\Delta 0$ met $15 \Delta 0$ ura3 $\Delta 0$ ), BY4741-derived mutants $c c c 2$ (MATa his3 31 leu2 $\Delta 0$ met15 $\Delta 0$ ura3 $\Delta 0$ YDR270w::kanMX4) and ctr1 (MATa his3 $\Delta 1$ leu2 $\Delta 0$ met15 $\Delta 0$ ura3 $\Delta 0$ YPR124w::kanMX4) were purchased from Open Biosystems (http://dharmacon.gelifesciences.com/openbiosystems). To generate the yeast expression vector, the full-length coding sequence of OsHMA4 was amplified from cDNA synthesized from TQ and LM and the CDS was ligated into the BamHI-EcoRI site of pYES2 vector. To generate the pYEC2-OsHMA4(TQ)-GFP and pYEC2-OsHMA4(LM)-GFP vectors, the OsHMA4 CDS was released from pYES2-OsHMA4(TQ) and pYES2-OsHMA4(LM) and ligate to the BamHI-EcoRI site of pYEC2/CT-GFP 52 . To generate the pYES2-OsHMA4(TQ)-GFP and pYES2-OsHMA4(LM)-GFP vectors, the coding sequence of GFP was released from pYEC2/CT-GFP and ligated to the XbaI site of pYES2-OsHMA4(TQ) and pYES2-OsHMA4(LM). The direction of GFP was confirmed by sequencing. The expression of OsHMA4 is under the control of a galactose-inducible promoter in these vectors. The resulting plasmids and empty vectors were transformed into various yeast strains using a Frozen-EZ Yeast Transformation II Kit (ZYMO Research). For complementation of the $c c c 2$ mutant, BY4741 and $c c c 2$ were transformed with pYES2 empty vector, pYES2-OsHMA4(TQ) or pYES2-OsHMA4(LM) and cultured at $30^{\circ} \mathrm{C}$ overnight in $3 \mathrm{ml}$ of SD-Ura media $\left(6.7 \mathrm{gl}^{-1}\right.$ yeast nitrogen base, $1.92 \mathrm{gl}^{-1}$ dropout mix without uracil) containing $2 \%$ (w/v) glucose. Cells were washed twice with $10 \mathrm{ml}$ sterile deionized water and the optical density at $630 \mathrm{~nm}$ adjusted to 0.2 with sterile distilled water. After sequential 10 -fold dilutions, $10 \mu \mathrm{l}$ of cell suspensions of each genotype were spotted on Fe-limited, Fe-sufficient and $\mathrm{Cu}$-sufficient media, respectively, and the plates incubated at $30^{\circ} \mathrm{C}$ for 3 days. Fe-limited media was prepared as previously described ${ }^{53}$, and contained $0.17 \%(\mathrm{w} / \mathrm{v})$ yeast nitrogen base without $\mathrm{CuSO}_{4}$ and $\mathrm{FeCl}_{3}$ (BIO 101 Systems), $0.2 \%$ (w/v) dropout mix without uracil, $2 \%(\mathrm{w} / \mathrm{v})$ galactose, $1 \%(\mathrm{w} / \mathrm{v})$ raffinose, $50 \mathrm{mM}$ MES $(\mathrm{pH}$ 6.1), $1 \mathrm{mM}$ 3-(2-pyridyl)-5, 6-bis (4-sulfophenyl)-1, 2, 4-triazine disodium salt (Ferrozine disodium salt; Sigma), $50 \mu \mathrm{M} \mathrm{Fe}\left(\mathrm{NH}_{4}\right)_{2}\left(\mathrm{SO}_{4}\right)_{2}, 1 \mu \mathrm{M} \mathrm{CuSO}_{4}$ and $2 \%(\mathrm{w} / \mathrm{v})$ agar. The Fe-sufficient and $\mathrm{Cu}$-sufficient media were modified from Fe-limited media by increasing the concentration of added $\mathrm{Fe}\left(\mathrm{NH}_{4}\right)_{2}\left(\mathrm{SO}_{4}\right)_{2}$ and $\mathrm{CuSO}_{4}$ to 350 and $500 \mu \mathrm{M}$, respectively, and the Ferrozine disodium salt was omitted. For the metal tolerance assays, diluted cell suspensions were prepared as above and spotted on the SD-Ura media containing $2 \%(\mathrm{w} / \mathrm{v})$ galactose, $1 \%(\mathrm{w} / \mathrm{v})$ raffinose and indicated metals. The growth of the BY4741 strain transformed with various plasmids in liquid SD-Ura media containing $\mathrm{Cu}$ was determined on $2 \mathrm{ml}$ 96-well deep plates. Overnight yeast cells were prepared as above and the optical density at $630 \mathrm{~nm}$ was adjusted to 0.5 with sterile distilled water. Fifty microliter of cell suspensions was added to $1 \mathrm{ml} \mathrm{SD-Ura} \mathrm{media} \mathrm{in} \mathrm{each} \mathrm{well} \mathrm{containing} 2 \%(\mathrm{w} / \mathrm{v})$ galactose, $1 \%(\mathrm{w} / \mathrm{v})$ raffinose and $0,2.5$ or $3 \mathrm{mM} \mathrm{CuSO}_{4}$. The plates were incubated at $30^{\circ} \mathrm{C}$ and shaken at 400 r.p.m. The optical density at $630 \mathrm{~nm}$ was determined at indicated time using a plate reader.

Homology modelling and sequence alignment. Homology modelling was conducted using the web-based SWISS-MODEL platform (http://swissmodel. expasy.org $)^{54}$. The crystal structure of a Legionella pneumophila P-type ATPase CopA (PDB ID: 3RFU) ${ }^{22}$ was used as a template. The models of OsHMA4 from TeQing and Lemont were built separately using the same default parameters. The model quality was evaluated using the structure assessment tools of the Swiss-Model workspace, including ANOLEA ${ }^{55}$, DFire $^{56}$ and QMEAN ${ }^{57}$. Structures were viewed using the DeepView/Swiss-PdbViewer 4.1 (http://www.expasy.org/ $\mathrm{spdbv} /)^{58}$. Multiple sequence alignments of HMA proteins were conducted in BioEdit software using the ClustalW method.

Genotyping of USDA rice core collection. The seeds of USDA Rice Core Collection were obtained from the Genetic Stocks Oryza (GSOR) Collection, USDA-ARS. In total, 1,349 accessions of the USDA Rice Core Collection were germinated and DNA was extracted for genotyping. Nine dCAPS markers were developed based on the non-synonymous polymorphisms in the coding sequence of OsHMA4 identified from 950 different rice genomes ${ }^{29}$. The primer sequences are listed in Supplementary Table 3. Five of these polymorphisms were confirmed to occur in the USDA Rice Core Collection. Among these five polymorphic sites, four of them had a minor allele frequency greater than 0.05 , which was further used to evaluate the contribution of OsHMA4 in controlling grain $\mathrm{Cu}$ in the population. A linear model was used to assess the proportion of variation in grain $\mathrm{Cu}$ in plants grown in flooded and unflooded conditions explained by the four polymorphic sites with a minor allele frequency $>0.05$. The linear model included each polymorphic site as explanatory variables. To account for potential nonindependence resulting from cryptic kinship between accessions, relative kinship among accessions was calculated using by SPAGeDi software ${ }^{59}$ based on 84 SSR markers $^{60}$, and the Loiselle coefficient ${ }^{61}$ was used to create the pair-wise kinship matrix. All negative values were set to zero ${ }^{62}$. A principal components analysis of the kinship matrix was performed and the eigenvectors of the first four principal components were included as explanatory variables in the linear model ${ }^{63}$. The first four principal components explained $87 \%$ of the variation in the kinship matrix. Only the accessions with the genotype available at all four polymorphic sites, genotyped by $84 \mathrm{SSR}$ markers, and grain $\mathrm{Cu}$ concentration were included in the analysis, resulting in a total of 1,241 accession under flooded condition and 1,210 accessions in unflooded condition.

The map image used in Fig. 6 was generated using R package rworldmap ${ }^{64}$ with data derived from Natural Earth v1.4.0 (http://www.naturalearthdata.com/).

Data availability. The authors declare that all data supporting the findings of this study are available within the article and its Supplementary Information files or are available on request from the corresponding authors.

\section{References}

1. Maksymiec, W. Effect of copper on cellular processes in higher plants. Photosynthetica 34, 321-342 (1997).

2. Burkhead, J. L., Reynolds, K. A., Abdel-Ghany, S. E., Cohu, C. M. \& Pilon, M. Copper homeostasis. New Phytol. 182, 799-816 (2009).

3. Pena, M. M. O., Lee, J. \& Thiele, D. J. A delicate balance: homeostatic control of copper uptake and distribution. J. Nutr. 129, 1251-1260 (1999).

4. Gulec, S. \& Collins, J. F. Molecular mediators governing iron-copper interactions. Annu. Rev. Nutr. 34, 95-116 (2014).

5. Allen, L., Benoist, B., Dary, O. \& Hurrell, R. in Guidelines on Food Fortification with Micronutrients (World Health Organization; Food and Agriculture Organization of the United Nations, 2006).

6. White, P. J. \& Broadley, M. R. Biofortification of crops with seven mineral elements often lacking in human diets - iron, zinc, copper, calcium, magnesium, selenium and iodine. New Phytol. 182, 49-84 (2009).

7. Collins, J. F. \& Klevay, L. M. Copper. Adv. Nutr. 2, 520-522 (2011).

8. Trumbo, P., Yates, A. A., Schlicker, S. \& Poos, M. Dietary reference intakes: vitamin $\mathrm{A}$, vitamin $\mathrm{K}$, arsenic, boron, chromium, copper, iodine, iron, manganese, molybdenum, nickel, silicon, vanadium, and zinc. J. Am. Diet. Assoc. 101, 294-301 (2001).

9. Zhao, F. J., Ma, Y. B., Zhu, Y. G., Tang, Z. \& McGrath, S. P. Soil contamination in China: current status and mitigation strategies. Environ. Sci. Technol. 49, 750-759 (2015).

10. Sancenon, V. et al. The Arabidopsis copper transporter COPT1 functions in root elongation and pollen development. J. Biol. Chem. 279, 15348-15355 (2004).

11. Yuan, M., Li, X., Xiao, J. \& Wang, S. Molecular and functional analyses of COPT/Ctr-type copper transporter-like gene family in rice. BMC Plant Biol. 11, 69 (2011).

12. Andres-Colas, N. et al. The Arabidopsis heavy metal P-type ATPase HMA5 interacts with metallochaperones and functions in copper detoxification of roots. Plant J. 45, 225-236 (2006).

13. Kobayashi, Y. et al. Amino acid polymorphisms in strictly conserved domains of a P-type ATPase HMA5 are involved in the mechanism of copper tolerance variation in Arabidopsis. Plant Physiol. 148, 969-980 (2008).

14. Shikanai, T., Muller-Moule, P., Munekage, Y., Niyogi, K. K. \& Pilon, M. PAA1, a P-type ATPase of Arabidopsis, functions in copper transport in chloroplasts. Plant Cell 15, 1333-1346 (2003).

15. Abdel-Ghany, S. E., Muller-Moule, P., Niyogi, K. K., Pilon, M. \& Shikanai, T. Two P-type ATPases are required for copper delivery in Arabidopsis thaliana chloroplasts. Plant Cell 17, 1233-1251 (2005).

16. Hirayama, T. et al. Responsive-to-antagonist1, a Menkes/Wilson disease-related copper transporter, is required for ethylene signaling in Arabidopsis. Cell 97, 383-393 (1999).

17. Woeste, K. E. \& Kieber, J. J. A strong loss-of-function mutation in RAN1 results in constitutive activation of the ethylene response pathway as well as a rosette-lethal phenotype. Plant Cell 12, 443-455 (2000).

18. Deng, F., Yamaji, N., Xia, J. \& Ma, J. F. A member of the heavy metal P-type ATPase OsHMA5 is involved in xylem loading of copper in rice. Plant Physiol. 163, 1353-1362 (2013).

19. Zheng, L., Yamaji, N., Yokosho, K. \& Ma, J. F. YSL16 is a phloem-localized transporter of the copper-nicotianamine complex that is responsible for copper distribution in rice. Plant Cell 24, 3767-3782 (2012).

20. Zhang, M. et al. Mapping and validation of quantitative trait loci associated with concentrations of 16 elements in unmilled rice grain. Theor. Appl. Genet. 127, 137-165 (2014).

21. Wang, M., Xu, Q., Yu, J. \& Yuan, M. The putative Arabidopsis zinc transporter ZTP29 is involved in the response to salt stress. Plant Mol. Biol. 73, 467-479 (2010).

22. Gourdon, P. et al. Crystal structure of a copper-transporting PIB-type ATPase. Nature 475, 59-64 (2011). 
23. Tuinstra, M. R., Ejeta, G. \& Goldsbrough, P. B. Heterogeneous inbred family (HIF) analysis: a method for developing near-isogenic lines that differ at quantitative trait loci. Theor. Appl. Genet. 95, 1005-1011 (1997).

24. Loudet, O., Gaudon, V., Trubuil, A. \& Daniel-Vedele, F. Quantitative trait loci controlling root growth and architecture in Arabidopsis thaliana confirmed by heterogeneous inbred family. Theor. Appl. Genet. 110, 742-753 (2005).

25. Xia, J. X., Yamaji, N., Kasai, T. \& Ma, J. F. Plasma membrane-localized transporter for aluminum in rice. Proc. Natl Acad. Sci. USA 107, 18381-18385 (2010).

26. Migocka, M., Posyniak, E., Maciaszczyk-Dziubinska, E., Papierniak, A. \& Kosieradzaka, A. Functional and biochemical characterization of cucumber genes encoding two copper ATPases CsHMA5.1 and CsHMA5.2. J. Biol. Chem. 290, 15717-15729 (2015).

27. Yuan, D. S. et al. The Menkes/Wilson disease gene homologue in yeast provides copper to a ceruloplasmin-like oxidase required for iron uptake. Proc. Natl Acad. Sci. USA 92, 2632-2636 (1995).

28. Ueno, D. et al. Gene limiting cadmium accumulation in rice. Proc. Natl Acad. Sci. USA 107, 16500-16505 (2010).

29. Huang, X. et al. Genome-wide association study of flowering time and grain yield traits in a worldwide collection of rice germplasm. Nat. Genet. 44, 32-39 (2012).

30. Pinson, S. R. M. et al. Worldwide genetic diversity for mineral element concentrations in rice grain. Crop Sci. 55, 294-311 (2015).

31. Huang, X. et al. A map of rice genome variation reveals the origin of cultivated rice. Nature 490, 497-501 (2012).

32. Wang, M. et al. The genome sequence of African rice (Oryza glaberrima) and evidence for independent domestication. Nat. Genet. 46, 982-988 (2014).

33. Fageria, N. K. et al. Chemistry of lowland rice soils and nutrient availability. Commun. Soil Sci. Plant Anal. 42, 1913-1933 (2011).

34. Morel, M. et al. AtHMA3, a P1B-ATPase allowing $\mathrm{Cd} / \mathrm{Zn} / \mathrm{Co} / \mathrm{Pb}$ vacuolar storage in Arabidopsis. Plant Physiol. 149, 894-904 (2009).

35. Chao, D. Y. et al. Genome-wide association studies identify heavy metal ATPase 3 as the primary determinant of natural variation in leaf cadmium in Arabidopsis thaliana. PLoS Genet. 8, e1002923 (2012).

36. Haydon, M. J. \& Cobbett, C. S. A novel major facilitator superfamily protein at the tonoplast influences zinc tolerance and accumulation in Arabidopsis. Plant Physiol. 143, 1705-1719 (2007).

37. Lee, S., Kim, Y. Y., Lee, Y. \& An, G. Rice P1B-type heavy-metal ATPase, OsHMA9, is a metal efflux protein. Plant Physiol. 145, 831-842 (2007).

38. Satoh-Nagasawa, N. et al. Mutations in rice (Oryza sativa) heavy metal ATPase 2 (OsHMA2) restrict the translocation of zinc and cadmium. Plant Cell Physiol. 53, 213-224 (2012).

39. Takahashi, R. et al. The OsHMA2 transporter is involved in root-to-shoot translocation of $\mathrm{Zn}$ and Cd in rice. Plant Cell Environ. 35, 1948-1957 (2012).

40. Yamaji, N., Xia, J., Mitani-Ueno, N., Yokosho, K. \& Ma, J. F. Preferential delivery of zinc to developing tissues in rice is mediated by P-type heavy metal ATPase OsHMA2. Plant Physiol. 162, 927-939 (2013).

41. Sasaki, A., Yamaji, N. \& Ma, J. F. Overexpression of OsHMA3 enhances $\mathrm{Cd}$ tolerance and expression of $\mathrm{Zn}$ transporter genes in rice. J. Exp. Bot. 65, 6013-6021 (2014)

42. Norton, G. J. et al. Genome wide association mapping of grain arsenic, copper, molybdenum and zinc in rice (Oryza sativa L.) grown at four international field sites. PLoS ONE 9, e89685 (2014).

43. Li, Z. K. et al. A 'defeated' rice resistance gene acts as a QTL against a virulent strain of Xanthomonas oryzae pv. oryzae. Mol. Gen. Genet. 261, 58-63 (1999).

44. Li, Z., Pinson, S. R., Stansel, J. W. \& Park, W. D. Identification of quantitative trait loci (QTLs) for heading date and plant height in cultivated rice (Oryza sativa L.). Theor. Appl. Genet. 91, 374-381 (1995).

45. Pinson, S. R. M. et al. Registration of a rice gene-mapping population consisting of 'TeQing'-into-'Lemont' backcross introgression lines. J. Plant Regist. 6, 128-135 (2012).

46. Jeon, J.-S. et al. T-DNA insertional mutagenesis for functional genomics in rice. Plant J. 22, 561-570 (2000).

47. Huang, X. Y. et al. A previously unknown zinc finger protein, DST, regulates drought and salt tolerance in rice via stomatal aperture control. Genes Dev. 23, 1805-1817 (2009).

48. Frame, B. R. et al. Agrobacterium tumefaciens-mediated transformation of maize embryos using a standard binary vector system. Plant Physiol. 129, 13-22 (2002).

49. Hiei, Y. \& Komari, T. Agrobacterium-mediated transformation of rice using immature embryos or calli induced from mature seed. Nat. Protoc. 3, 824-834 (2008).

50. Clough, S. J. \& Bent, A. F. Floral dip: a simplified method for Agrobacteriummediated transformation of Arabidopsis thaliana. Plant J. 16, 735-743 (1998).

51. Berthomieu, P. et al. Functional analysis of AtHKT1 in Arabidopsis shows that $\mathrm{Na}^{+}$recirculation by the phloem is crucial for salt tolerance. EMBO J. 22, 2004-2014 (2003).
52. Craciun, A. R. et al. Variation in HMA4 gene copy number and expression among Noccaea caerulescens populations presenting different levels of $\mathrm{Cd}$ tolerance and accumulation. J. Exp. Bot. 63, 4179-4189 (2012).

53. Southron, J. L., Basu, U. \& Taylor, G. J. Complementation of Saccharomyces cerevisiae ccc2 mutant by a putative P-1B-ATPase from Brassica napus supports a copper-transporting function. FEBS Lett. 566, 218-222 (2004).

54. Arnold, K., Bordoli, L., Kopp, J. \& Schwede, T. The SWISS-MODEL workspace: a web-based environment for protein structure homology modelling. Bioinformatics 22, 195-201 (2006).

55. Melo, F. \& Feytmans, E. Assessing protein structures with a non-local atomic interaction energy. J. Mol. Biol. 277, 1141-1152 (1998).

56. Zhou, H. \& Zhou, Y. Distance-scaled, finite ideal-gas reference state improves structure-derived potentials of mean force for structure selection and stability prediction. Protein Sci. 11, 2714-2726 (2002).

57. Benkert, P., Biasini, M. \& Schwede, T. Toward the estimation of the absolute quality of individual protein structure models. Bioinformatics 27, 343-350 (2011)

58. Guex, N. \& Peitsch, M. C. SWISS-MODEL and the Swiss-PdbViewer: an environment for comparative protein modeling. Electrophoresis 18, 2714-2723 (1997).

59. Hardy, O. J. \& Vekemans, X. SPAGeDi: a versatile computer program to analyse spatial genetic structure at the individual or population levels. Mol. Ecol. Notes 2, 618-620 (2002).

60. Yan, W. G., Agrama, H., Jia, M., Fjelistrom, R. \& McClung, A. Geographic description of genetic diversity and relationships in the USDA rice world collection. Crop Sci. 50, 2406-2417 (2010).

61. Loiselle, B. A., Sork, V. L., Nason, J. \& Graham, C. Spatial genetic structure of a tropical understory shrub, Psychotria officinalis (Rubiaceae). Am. J. Bot. 82, 1420-1425 (1995).

62. Yu, J. et al. A unified mixed-model method for association mapping that accounts for multiple levels of relatedness. Nat. Genet. 38, 203-208 (2006).

63. Price, A. L. et al. Principal components analysis corrects for stratification in genome-wide association studies. Nat. Genet. 38, 904-909 (2006).

64. South, A. rworldmap. a new $\mathrm{r}$ package for mapping global data. $R$ J. 3, 35-43 (2011)

\section{Acknowledgements}

We thank B. Lahner, E. Yakubova and S. Rikiishi for ICP-MS analysis, N. Komiyama, Iowa State University Plant Transformation Facility and Prashant Hosmani for generation of transgenic rice, K. Wang for providing pTF101.1 vector and N. Verbruggen for providing pYES2 and pYEC2/CT-GFP vectors. We also thank Rice T-DNA Insertion Sequence Database center for providing the T-DNA insertion line and X. Wang, $\mathrm{T}$. Zheng and $\mathrm{Z}$. Li for accessing $3 \mathrm{~K}$ rice genome sequence, and Graeme Paton for helpful discussions on $\mathrm{Cu}$ bioavailability in water-logged soils. This research was supported by a Grant-in-Aid for Specially promoted Research (JSPS KAKENHI Grant Number 16H06296 to J.F.M), and the US National Science Foundation, Plant Genome Research Program (Grant \#IOS 0701119 to D.E.S., M.L.G. and S.R.M.P.)

\section{Author contributions}

X.-Y.H., F.D., D.E.S., and J.F.M. designed the research; X.-Y.H., F.D., N.Y., S.R.M.P., M.F.-K., J.D. and M.L.G. performed the research; X.-Y.H., F.D., A.D., D.E.S. and J.F.M. analysed the data; and X.-Y.H., D.E.S. and J.F.M. wrote the paper.

\section{Additional information}

Accession codes: The sequences of OsHMA4 from TeQing and Lemont have been deposited in GenBank nucleotide database under the accession code KU168831 and KU168832, respectively.

Supplementary Information accompanies this paper at http://www.nature.com/ naturecommunications

Competing financial interests: The authors declare no competing financial interests.

Reprints and permission information is available online at http://npg.nature.com/ reprintsandpermissions/

How to cite this article: Huang, X.-Y. et al. A heavy metal P-type ATPase OsHMA4 prevents copper accumulation in rice grain. Nat. Commun. 7:12138 doi: $10.1038 /$ ncomms12138 (2016).

This work is licensed under a Creative Commons Attribution 4.0 International License. The images or other third party material in this article are included in the article's Creative Commons license, unless indicated otherwise in the credit line; if the material is not included under the Creative Commons license, users will need to obtain permission from the license holder to reproduce the material To view a copy of this license, visit http://creativecommons.org/licenses/by/4.0/

(C) The Author(s) 2016 